\title{
APPROXIMATE SOLUTIONS OF GENERALIZED RIEMANN PROBLEMS FOR NONLINEAR SYSTEMS OF HYPERBOLIC CONSERVATION LAWS
}

\author{
CLAUS R. GOETZ AND ARMIN ISKE
}

\begin{abstract}
We study analytical properties of the Toro-Titarev solver for generalized Riemann problems (GRPs), which is the heart of the flux computation in ADER generalized Godunov schemes. In particular, we compare the ToroTitarev solver with a local asymptotic expansion developed by LeFloch and Raviart. We show that for nonlinear scalar problems in 1D the Toro-Titarev solver reproduces the truncated Taylor series expansion of LeFloch-Raviart exactly, whereas for nonlinear systems the Toro-Titarev solver introduces an error whose size depends on the height of the jump in the initial data. Thereby, our analysis answers open questions concerning the justification of simplifying steps in the Toro-Titarev solver. We illustrate our results by giving the full analysis for a nonlinear 2-by-2 system and numerical results for shallow water equations and a system from traffic flow.
\end{abstract}

\section{INTRODUCTION}

The classical Godunov method approximates the solution of a hyperbolic conservation law by a piecewise constant function and then solves local Riemann problems exactly to evolve that data. Clearly, piecewise constant approximation limits the order of accuracy, and so the natural question to ask is: Can we construct more accurate schemes by using piecewise smooth functions, e.g., polynomials of higher degree, rather than piecewise constant functions? To construct a generalized Godunov scheme we need to solve the initial value problem with piecewise smooth data. We call any Cauchy problem with piecewise smooth initial data (that may be discontinuous at the origin) generalized Riemann problem (GRP).

While classical Riemann problems (RPs) can be solved exactly for many relevant cases, generalized Riemann problems (GRPs) are much more complicated. In the case of nonlinear systems, analytical expressions for the solution of GRPs are usually not available. Toro and Titarev [37] have proposed a computational method, ToroTitarev solver, for approximately solving the GRP. While the Toro-Titarev solver has been used quite successfully in a wide range of applications (see [1, 15, 25, 30, 33, 35]), only very few contributions concerning the solver's theoretical properties have been provided so far. In fact, it is the demand for a more rigorous analysis on the properties of the Toro-Titarev solver that has motivated this paper.

Received by the editor September 4, 2013 and, in revised form, April 24, 2014.

2010 Mathematics Subject Classification. Primary 65M08; Secondary 65D15, 58J45, 35L65.

Key words and phrases. Hyperbolic conservation laws, generalized Riemann problems, ADER methods. 
Starting with the pioneering work of Kolgan [16] and van Leer [38, piecewise linear reconstruction in space has become a commonly used tool for improving accuracy over the Godunov scheme. An early example for the use of higher order polynomials is the piecewise parabolic method (PPM) of Collela and Woodward [1]. and, as pointed out in [8], the numerical flux proposed by Harten, Engquist, Osher and Chakravarthy in their seminal work on ENO methods 13 can be interpreted in a generalized Godunov framework. However, the scheme that has, from a conceptual point of view, the most in common with what we discuss in this paper is the GRP scheme of Ben-Artzi and Falcovitz [4, 5].

A state-of-the-art variant of the generalized Godunov approach is the ADER scheme 29, 34]. The ADER scheme relies on a high order WENO-reconstruction 3, 14, 23. from cell-averages and the solution of GRPs at the cell-interfaces. To solve GRPs numerically, Toro and Titarev 37] proposed to build a Taylor approximation of the solution whose coefficients are computed by solving a sequence of classical RPs.

In this paper, we focus on hyperbolic systems in conservation form in one spatial dimension, but the ADER approach can be extended to a much broader set of problems; see e.g. [1, 15, 25, 30, 33, 35]. Stability and the order of accuracy can be verified numerically; see [31] and references therein. However, it was reported by Castro and Toro [8] that the Toro-Titarev solver in 37] encounters difficulties for nonlinear systems with large jumps in the initial data. This phenomenon has not been explained yet.

We analyse the Toro-Titarev solver by comparing it to the local asymptotic expansion for the solution of the GRP that was constructed by LeFloch and Raviart [20]. In the following we call this method the LeFloch-Raviart solver. We show analytically that both methods yield the same truncated Taylor series expansion for nonlinear scalar problems, whereas there is a difference for nonlinear systems. Both methods formally construct the same Taylor series expansion, but in the case of nonlinear systems the Toro-Titarev solver uses an approximation to spatial derivatives at the origin that differs from the values obtained in the LeFloch-Raviart solver through the Rankine-Hugoniot conditions. Moreover, that difference can be clearly tracked back to the size of the jump in the initial data.

The outline of this paper is as follows. In Section 2 we set up the analytic framework and review well-known results on the structure of solutions to classical RPs and GRPs. We explain generalized Godunov schemes in Section 3, before we discuss the Toro-Titarev solver in Section 4 . Key steps for the construction of the LeFloch-Raviart solver are presented in Section 5. In Section 6 we compare the resulting approximations to the solution of the GRP. We finally apply the two solution strategies in Section 7 , by using a $2 \times 2$ system arising from two-component chromatography to illustrate the analytical techniques and provide numerical examples for shallow water equations and for a system from traffic flow.

\section{Classical and generalized Riemann problems}

Consider a nonlinear $m \times m$ system of hyperbolic conservation laws,

$$
\frac{\partial}{\partial t} u+\frac{\partial}{\partial x} f(u)=0, \quad x \in \mathbb{R}, t>0, \quad u(x, t) \in \mathcal{U} \subset \mathbb{R}^{m},
$$

where $\mathcal{U} \subset \mathbb{R}^{m}$ is an open and convex subset and $f: \mathcal{U} \rightarrow \mathbb{R}^{m}$ is a smooth function. The classical Riemann problem (RP) is the Cauchy problem for (2.1) with initial 
data

$$
u(x, 0)= \begin{cases}\hat{u}_{L}^{0}, & \text { if } x<0, \\ \hat{u}_{R}^{0}, & \text { if } x>0 .\end{cases}
$$

The initial data is piecewise constant and given by the vectors $\hat{u}_{L}^{0}, \hat{u}_{R}^{0} \in \mathcal{U}$. Assume (2.1) to be strictly hyperbolic, i.e., the Jacobian $A(u)=D f(u)$ has $m$ distinct real eigenvalues

$$
\lambda_{1}(u)<\lambda_{2}(u)<\cdots<\lambda_{m}(u) \quad \text { for all } u \in \mathcal{U} .
$$

We choose bases of left and right eigenvectors of $A(u)$, i.e., bases of $\mathbb{R}^{m}$, $\left\{\ell_{1}(u), \ldots, \ell_{m}(u)\right\}$, and $\left\{r_{1}(u), \ldots, r_{m}(u)\right\}$, such that for all $u \in \mathcal{U}$ we have

$$
A(u) r_{i}(u)=\lambda_{i}(u) r_{i}(u), \quad \ell_{i}(u)^{T} A(u)=\lambda_{i}(u) \ell_{i}(u)^{T}, \quad i=1, \ldots, m .
$$

The eigenvectors are normalized to

$$
\left|r_{i}(u)\right|=1, \quad \ell_{j}(u)^{T} r_{i}(u)=\left\{\begin{array}{ll}
1, & i=j, \\
0, & i \neq j,
\end{array} \text { for all } u \in \mathcal{U}\right.
$$

We restrict our analysis to systems for which every characteristic field is either genuinely nonlinear in the sense of Lax [19],

$$
\nabla \lambda_{i}(u)^{T} r_{i}(u) \neq 0 \quad \text { for all } u \in \mathcal{U},
$$

or linearly degenerate, i.e.,

$$
\nabla \lambda_{i}(u)^{T} r_{i}(u) \equiv 0 .
$$

Under these assumptions we have the following well-known result: Given two states $\hat{u}_{L}^{0}, \hat{u}_{R}^{0} \in \mathcal{U}$ with $\left|\hat{u}_{R}^{0}-\hat{u}_{L}^{0}\right|>0$ sufficiently small, the classical RP (2.1), (2.2) has a unique entropy admissible weak solution $u(x, t)=u^{0}(x / t)$ that is self-similar and consists of $m+1$ constant states

$$
\hat{u}_{L}^{0}=u_{0}, u_{1}, \ldots, u_{m-1}, u_{m}=\hat{u}_{R}^{0},
$$

separated by rarefaction waves, shock waves or contact discontinuities. For a comprehensive analysis of the classical RP and the properties of its solution, see e.g. [26].

Next, assume that the initial data

$$
u(x, 0)= \begin{cases}\hat{u}_{L}(x), & \text { if } x<0, \\ \hat{u}_{R}(x), & \text { if } x>0\end{cases}
$$

with $\hat{u}_{L}, \hat{u}_{R}: \mathbb{R} \rightarrow \mathcal{U}$, is piecewise smooth but discontinuous at $x=0$. The Cauchy problem (2.1), (2.4) is called the generalized Riemann problem (GRP). We let $\hat{u}_{L}^{0}=\hat{u}_{L}(0)$ and $\hat{u}_{R}^{0}=\hat{u}_{R}(0)$. It is well known (see [22, 27]) that for sufficiently small $\left|\hat{u}_{R}^{0}-\hat{u}_{L}^{0}\right|>0$, there exists a neighbourhood around the origin in which (2.1), (2.4) has a unique entropy admissible weak solution.

Moreover, for sufficiently small $T>0$, the strip $\mathbb{R} \times[0, T)$ can be decomposed into $m+1$ open domains of smoothness $D_{i}, 0 \leq i \leq m$, separated by smooth curves $\gamma_{j}(t)$ passing throuh the origin, or by rarefaction zones with boundaries $\underline{\gamma}_{j}(t), \bar{\gamma}_{j}(t)$, where $\underline{\gamma}_{j}(t), \bar{\gamma}_{j}(t)$ are smooth characteristic curves passing through the origin. More precisely, we have curves $\gamma_{j}(t)$ and rarefaction zones

$$
R_{j}=\left\{(x, t) \in \mathbb{R} \times[0, T) \mid \underline{\gamma}_{j}(t)<x<\bar{\gamma}_{j}(t)\right\} .
$$


For $\gamma_{j}(t)$, we let $\underline{\gamma}_{j}(t)=\bar{\gamma}_{j}(t)=\gamma_{j}(t)$ for all $t \in[0, T)$. Then, we can write

$D_{0}=\left\{(x, t) \in \mathbb{R} \times[0, T) \mid x<\underline{\gamma}_{1}(t)\right\}, \quad D_{m}=\left\{(x, t) \in \mathbb{R} \times[0, T) \mid \bar{\gamma}_{m}(t)<x\right\}$,

$D_{i}=\left\{(x, t) \in \mathbb{R} \times[0, T) \mid \bar{\gamma}_{i}(t)<x<\underline{\gamma}_{i+1}(t)\right\}, \quad 1 \leq i \leq m-1$.

The solution $u$ is smooth inside each domain $D_{i}$ and inside each rarefaction zone $R_{j}$. Moreover, $u$ has a shock or contact discontinuity across each curve $x=\gamma_{j}(t)$ and is continuous across the characteristic curves $x=\underline{\gamma}_{j}(t), x=\bar{\gamma}_{j}(t)$.

The solution of the GRP and the solution of the corresponding classical RP with the initial states $\hat{u}_{L}^{0}=\hat{u}_{L}(0)$ and $\hat{u}_{R}^{0}=\hat{u}_{R}(0)$ have a similar wave structure, at least for small time $t>0$. That is, if the $j$-wave in the solution of the classical RP is a shock wave, a contact discontinuity or a rarefaction wave, then the corresponding $j$-wave in the GRP is of the same respective type.

In this paper, we focus on the case where the solution contains only shock waves and contact discontinuities. In this case, the connection between the wave structures can be described more precisely: Denote the constant states in the solution of the RP with initial data $\hat{u}_{L}^{0}, \hat{u}_{R}^{0}$ by $u_{i}^{0}$, for $i=0, \ldots, m$, and the wave speeds by $\sigma_{j}^{0}, j=1, \ldots, m$. Then, the curves $\gamma_{j}$ satisfy

$$
\gamma_{j}(0)=0 \quad \text { and } \quad \lim _{t \rightarrow 0} \dot{\gamma}_{j}(t)=\sigma_{j}^{0}, \quad \text { for } j=1, \ldots, m,
$$

and the solution $u$ of the GRP satisfies within each domain of smoothness $D_{i}$ the convergence

$$
\lim _{\substack{t \rightarrow 0 \\(x, t) \in D_{i}}} u(x, t)=u_{i}^{0} \quad \text { for } i=0, \ldots, m .
$$

We remark that the solution of GRPs is a popular subject of ongoing research. Quite recently, special emphasis has been placed on the global existence and the structural stability of solutions. We refer to [9, 10, 17, 18] and references therein for an up-to-date account on the solution of GRPs. For the following analysis in this paper, we can rely on available results on the local existence and on the local structural stability.

\section{Generalized Godunov schemes}

To solve the Cauchy problem

$$
\frac{\partial}{\partial t} u+\frac{\partial}{\partial x} f(u)=0, \quad x \in \mathbb{R}, t>0, \quad u(x, 0)=\hat{u}(x),
$$

numerically, we extend the classical Godunov finite volume scheme, by assuming a uniform grid

$$
x_{i+1 / 2}=(i+1 / 2) \Delta x, \quad t^{n}=n \Delta t \quad i \in \mathbb{Z}, n \in \mathbb{N},
$$

for simplicity, where $\Delta x>0, \Delta t>0$.

A generalized Godunov scheme consists of the following steps: Starting with cell averages

$$
\bar{u}_{i}^{0}=\frac{1}{\Delta x} \int_{x_{i-1 / 2}}^{x_{i+1 / 2}} \hat{u}(x) d x, \quad i \in \mathbb{Z},
$$

for any time step $n=0,1, \ldots$, given the values $\left\{\bar{u}_{i}^{n}\right\}_{i \in \mathbb{Z}}$, perform the following steps: 
(1) Find a piecewise smooth, conservative reconstruction. That is, compute a function $\hat{u}^{n}: \mathbb{R} \rightarrow \mathcal{U}$ such that for all $i \in \mathbb{Z}$ we have

$\hat{u}_{i}^{n}=\left.\hat{u}^{n}\right|_{\left[x_{i-1 / 2}, x_{i+1 / 2}\right]}$ is smooth, $\quad$ and $\frac{1}{\Delta x} \int_{x_{i-1 / 2}}^{x_{i+1 / 2}} \hat{u}^{n}(x) d x=\bar{u}_{i}^{n}$.

For ADER schemes this is usually done by a WENO-reconstruction [3, 14, 23], such that each $\hat{u}_{i}^{n}$ is a polynomial of degree $r-1$, where $r>1$ is a given integer.

(2) Use the function $\hat{u}^{n}$ as initial data, i.e., pose the Cauchy problem

$$
\begin{array}{lr}
\frac{\partial}{\partial t} u+\frac{\partial}{\partial x} f(u)=0, & x \in \mathbb{R}, t>0, \\
u(x, 0)=\hat{u}_{i}^{n}(x), & x \in\left[x_{i-1 / 2}, x_{i+1 / 2}\right], i \in \mathbb{Z} .
\end{array}
$$

Solve this problem exactly and evolve the data for one time step. Denote by $\mathcal{E}$ the exact entropy evolution operator associated with (3.1) and by $\Delta t-$ the limit $t \rightarrow \Delta t, t<\Delta t$. We compute $\mathcal{E}(\Delta t-) \hat{u}^{n}$.

(3) Update the cell averages by

$$
\bar{u}_{i}^{n+1}=\frac{1}{\Delta x} \int_{x_{i-1 / 2}}^{x_{i+1 / 2}} \mathcal{E}(\Delta t-) \hat{u}^{n}(x) d x .
$$

In a finite volume framework we can perform evolution and averaging in one step by the update

$$
\bar{u}_{i}^{n+1}=\bar{u}_{i}^{n}-\frac{\Delta t}{\Delta x}\left(\bar{f}_{i+1 / 2}^{n}-\bar{f}_{i-1 / 2}^{n}\right) .
$$

Here, $\bar{f}_{i+1 / 2}^{n}$ is the exact averaged flux through the cell interface $x_{i+1 / 2}$ during one time step:

$$
\bar{f}_{i+1 / 2}^{n}=\frac{1}{\Delta t} \int_{0}^{\Delta t} f\left(\mathcal{E}(\tau) \hat{u}^{n}\left(x_{i+1 / 2}\right)\right) d \tau,
$$

and $\tau=t-t^{n}$ is the local time. However, computing the exact integral (3.2) may be exceedingly complicated, if not impossible. Therefore, we are looking for an approximation to (3.2) based on an approximate solution of the GRP.

\section{The Toro-Titarev solver for the Generalized Riemann Problem}

We describe the method of solution for the GRP proposed by Toro and Titarev [37], based on a state expansion approach. That is, we use a Taylor series expansion of order $r$ in time of the solution around $\tau=0$ right at the cell interface $x_{i+1 / 2}$,

$$
u\left(x_{i+1 / 2}, \tau\right) \approx u\left(x_{i+1 / 2}, 0+\right)+\sum_{k=1}^{r-1} \frac{\partial^{k} u}{\partial t^{k}}\left(x_{i+1 / 2}, 0+\right) \frac{\tau^{k}}{k !} .
$$

Note that while the solution $u$ may be discontinuous, the function $u\left(x_{i+1 / 2}, \cdot\right)$ for a fixed point $x_{i+1 / 2}$ in space as a function of time is smooth, provided that $\tau>0$ is small enough.

If we can solve the GRP and give a meaning to the time derivatives in (4.1), the easiest way to define a numerical flux is to approximate the time-integral in (3.2) by a Gaussian quadrature,

$$
f_{i+1 / 2}^{n}=\sum_{\gamma=1}^{N} \omega_{\gamma} f\left(u\left(x_{i+1 / 2}, \tau_{\gamma}\right)\right)
$$


where $\omega_{\gamma}, \tau_{\gamma}$ are suitable weights and nodes and $N$ is the number of nodes, which is chosen according to the desired accuracy. The values $u\left(x_{i+1 / 2}, \tau_{\gamma}\right)$ are computed by (4.1). For a discussion of more refined numerical fluxes in the ADER context, see 32,36 .

The key idea in the method of Toro and Titarev [37] is to reduce the solution of the GRP to a sequence of classical RPs. To find the desired value $u\left(x_{i+1 / 2}, 0+\right)$ we take the extrapolated values $\hat{u}_{L}^{0}=\hat{u}_{L}\left(x_{i-1 / 2}-\right)$ and $\hat{u}_{R}^{0}=\hat{u}_{R}\left(x_{i+1 / 2}+\right)$ to solve the classical RP:

$$
\begin{array}{ll}
\frac{\partial}{\partial t} u+\frac{\partial}{\partial x} f(u)=0, & x \in \mathbb{R}, t>0, \\
u(x, 0)= \begin{cases}\hat{u}_{L}^{0} & \text { if } x<x_{i+1 / 2}, \\
\hat{u}_{R}^{0} & \text { if } x>x_{i+1 / 2} .\end{cases}
\end{array}
$$

As described in Section 2, this problem has a self-similar entropy solution that we denote by $u^{0}\left(\left(x-x_{i+1 / 2}\right) / \tau\right)$. The leading term of the expansion (4.1) is then given by $u\left(x_{i+1 / 2}, 0+\right)=u^{0}(0)$. We call this the Godunov state of (4.2), (4.3). For nonlinear systems of conservation laws, computing the solution of the RP may be difficult, so we might need to employ a numerical (approximative) Riemann solver (see [32]) to compute the leading term. However, in this paper we are mainly interested in the analytical aspects of the scheme, so we assume all states and wave speeds in the solution of the RP (4.2), (4.3) can be computed exactly.

For higher order terms we formally perform a Cauchy-Kowalewskaya procedure to express all time derivatives as functions of spatial derivatives up to the same order. That is, we use a recursive mapping

$$
\frac{\partial^{k} u}{\partial t^{k}}=\Phi^{k}\left(u, \frac{\partial u}{\partial x}, \ldots, \frac{\partial^{k} u}{\partial x^{k}}\right), \quad k=0, \ldots, r-1, \quad \text { with } \Phi^{0}(u)=u .
$$

Since the classical Cauchy-Kowalewskaya theorem assumes analytical initial data, it does not apply to the case of piecewise smooth data. But to illustrate the basic ideas, assume $u$ was smooth. In that case, the equations in the following can be obtained by simple manipulations of derivatives.

We can compute the expansion (4.1) via $\Phi^{k}$, provided that we can find the spatial derivatives

$$
u^{k}(0)=\lim _{\substack{x \rightarrow x_{i+1 / 2} \\ t \rightarrow 0+}} \frac{\partial^{k} u}{\partial x^{k}}(x, t) .
$$

To do so, we use the one-sided derivatives

$$
\hat{u}_{L}^{k}=\lim _{x \rightarrow x_{i+1 / 2,-}} \frac{\partial^{k} \hat{u}_{L}}{\partial x^{k}}(x), \quad \hat{u}_{R}^{k}=\lim _{x \rightarrow x_{i+1 / 2,+}} \frac{\partial^{k} \hat{u}_{R}}{\partial x^{k}}(x) .
$$

These values can be used as initial conditions for classical RPs. As regards the evolution equations for the spatial derivatives, we can rely on the following result: Let $u$ be a smooth solution of (3.1), and for $k \geq 1$, denote the $k$-th spatial derivative of $u$ by $u^{k}$. Then, all $u^{k}$ satisfy a semi-linear hyperbolic equation of the form

$$
\frac{\partial}{\partial t} u^{k}+A(u) \frac{\partial}{\partial x} u^{k}=H^{k}\left(u, u^{1}, \ldots, u^{k}\right)
$$

where $A(u)=D f(u)$ is the Jacobian of the flux and the function $H^{k}$ depends only on $u, u^{1}, \ldots, u^{k}$.

We remark that for smooth $u$, (4.4) can be obtained by straightforward computation. For the sake of brevity, however, we omit the details here. Moreover, it 
is easy to see that in the linear case, where $A(u) \equiv A$ is a constant matrix, the function $H^{k}$ vanishes identically. Although we can derive (4.4) for smooth $u$, we do not have a rigorous analysis yet to see whether these equations can also be used for discontinuous solutions.

We simplify the problem (4.4) in two ways: First, we neglect the source terms and second, we linearize the equations:

$$
\begin{aligned}
& \frac{\partial}{\partial t} u^{k}+A_{L R} \frac{\partial}{\partial x} u^{k}=0, \quad \text { for } k=1, \ldots, r-1, \\
& u^{k}(x, 0)= \begin{cases}\hat{u}_{L}^{k}, & \text { if } x<x_{i+1 / 2}, \\
\hat{u}_{R}^{k}, & \text { if } x>x_{i+1 / 2} .\end{cases}
\end{aligned}
$$

Here $A_{L R}=A\left(u\left(x_{i+1 / 2}, 0+\right)\right)$. Then, the self-similar solutions $u^{k}$ of these linear problems can be computed easily. Note that for all $k$ we have the same $A_{L R}$.

Finally, we approximate the solution $u$ along the $t$-axis by the truncated Taylor expansion

$$
u\left(x_{i+1 / 2}, \tau\right) \approx u^{0}(0)+\sum_{k=1}^{r-1} \Phi^{k}\left(u^{0}, u^{1}, \ldots, u^{k}\right)(0) \frac{\tau^{k}}{k !}
$$

\section{The LeFloch-Raviart solver for the Generalized Riemann Problem}

5.1. Basics. We describe how to construct a local power series expansion for the solution of the GRP. The main source for our work is the expansion constructed by LeFloch and Raviart [20. See [6] for an application of this techniques to the Euler equations of gas dynamics. Related approaches are discussed in 12 and [21]. Another somewhat different approach to asymptotic expansion for the Euler equations is given in 24. We discuss the local properties of the solution of the GRP

$$
\begin{array}{ll}
\frac{\partial}{\partial t} u+\frac{\partial}{\partial x} f(u)=0, & x \in \mathbb{R}, t>0, \\
u(x, 0)= \begin{cases}\hat{u}_{L}(x), & \text { if } x<0, \\
\hat{u}_{R}(x), & \text { if } x>0 .\end{cases}
\end{array}
$$

Our goal is to construct an asymptotic expansion of the form

$$
u(x, t)=\sum_{k \geq 0} t^{k} q^{k}(\xi)
$$

with $\xi=x / t$. This is possible in any domain of smoothness $D_{i}$, by simply taking a Taylor series expansion of the solution $u$ in that domain. So every $q^{k}$ is a polynomial of degree $k$. We return to this point in Section 6 .

It can be shown that such a series expansion can also be constructed inside a rarefaction zone $R$. However, for our numerical scheme we only need detailed information about the solution along the line segment $\{x=0\} \times[0, \Delta t]$ (in local coordinates). We assume that the solution does not contain a transonic rarefaction wave. In that case, the solution along that line segment is given by some function $u_{i *}$ inside a domain of smoothness $D_{i *}, 0 \leq i * \leq m$, and we do not need the explicit construction of the expansion inside a rarefaction zone.

Roughly speaking, the construction can be summarized as follows: Take a Taylor expansion wherever the solution $u$ is smooth and then investigate the jump conditions at the boundaries of the domains of smoothness. As we are looking for an 
expansion in terms of self-similar functions, it is useful to change the variables and work with $\xi=x / t$. We set $\tilde{u}(\xi, t)=u(\xi t, t)$ and check that

$$
\frac{\partial}{\partial x}=\frac{1}{t} \frac{\partial}{\partial \xi}, \quad \frac{\partial u}{\partial t}=\frac{\partial \tilde{u}}{\partial t}-\frac{\xi}{t} \frac{\partial \tilde{u}}{\partial \xi}
$$

For illustration we will give more details for scalar problems and $2 \times 2$ systems of conservation laws,

$$
u=\left(\begin{array}{c}
v \\
w
\end{array}\right) \in \mathcal{U} \subset \mathbb{R}^{2}, \quad f: \mathcal{U} \rightarrow \mathbb{R}^{2}, \quad f(u)=\left(\begin{array}{c}
f_{1}(v, w) \\
f_{2}(v, w)
\end{array}\right)
$$

in which case we denote the expansion by

$$
v(x, t)=\sum_{k \geq 0} t^{k} v^{k}(\xi), \quad w(x, t)=\sum_{k \geq 0} t^{k} w^{k}(\xi), \quad q^{k}(\xi)=\left(\begin{array}{c}
v^{k}(\xi) \\
w^{k}(\xi)
\end{array}\right) .
$$

We give the full detail for the construction of the expansion up to quadratic terms. First order terms were presented in [6] for the Euler equations, but there seems to be no explicit computation of higher order terms available in the literature.

5.2. Step I: Derivation of the differential equations. At first we derive a series of ordinary differential equations satisfied by the functions $q^{k}$ in (5.1). We change the variables according to (5.2) and the conservation law becomes

$$
t \frac{\partial}{\partial t} \tilde{u}-\xi \frac{\partial}{\partial \xi} \tilde{u}+\frac{\partial}{\partial \xi} f(\tilde{u}(\xi, t))=0
$$

Observe that the expansion

$$
\tilde{u}(\xi, t)=\sum_{k \geq 0} t^{k} q^{k}(\xi)
$$

gives

$$
t \frac{\partial \tilde{u}}{\partial t}-\xi \frac{\partial \tilde{u}}{\partial \xi}=-\xi \frac{d q^{0}}{d \xi}+\sum_{k \geq 1} t^{k}\left(k q^{k}-\xi \frac{d q^{k}}{d \xi}\right)
$$

Inserting this expansion into the flux function $f$ yields

$$
f(\tilde{u}(\xi, t))=f\left(q^{0}\right)+\sum_{k \geq 1} t^{k}\left(A\left(q^{0}\right) u^{k}+f^{k}\left(Q^{k-1}\right)\right) .
$$

Here, the function $f^{k}$ depends only on the previous terms $Q^{k-1}=\left(q^{0}, \ldots, q^{k-1}\right)$. We get $f^{k}$ by a Taylor expansion of the flux in powers of $t$, such that $f^{k}$ accounts for all terms in that expansion belonging to $t^{k}$ that do not depend on $q^{k}$, i.e., all but $A\left(q^{0}\right) q^{k}$. This will be our standard trick in the following analysis, so we discuss the method in somewhat more detail. 
At first, consider the expansion of the flux around $t=0$ for the scalar case:

$$
\begin{aligned}
f\left(\sum_{k \geq 0} t^{k} q^{k}(\xi)\right)= & f\left(q^{0}\right)+\left.t \frac{\partial}{\partial t} f\left(\sum_{k \geq 0} t^{k} q^{k}(\xi)\right)\right|_{t=0}+\left.\frac{t^{2}}{2} \frac{\partial^{2}}{\partial t^{2}} f\left(\sum_{k \geq 0} t^{k} q^{k}(\xi)\right)\right|_{t=0}+\ldots \\
= & f\left(q^{0}\right)+\left.\left.t f^{\prime}\left(\sum_{k \geq 0} t^{k} q^{k}(\xi)\right)\right|_{t=0}\left(\sum_{k \geq 0} k t^{k-1} q^{k}(\xi)\right)\right|_{t=0} \\
& +\frac{t^{2}}{2}\left\{\left.f^{\prime \prime}\left(\sum_{k \geq 0} t^{k} q^{k}(\xi)\right)\right|_{t=0}\left(\sum_{k \geq 0} k t^{k-1} q^{k}(\xi)\right)^{2}\right\} \\
& +\frac{t^{2}}{2}\left\{\left.\left.f^{\prime}\left(\sum_{k \geq 0} t^{k} q^{k}(\xi)\right)\right|_{t=0}\left(\sum_{k \geq 0} k(k-1) t^{k-2} q^{k}(\xi)\right)\right|_{t=0}\right\}+\ldots
\end{aligned}
$$

So we have

$f(\tilde{u}(\xi, t))=f\left(q^{0}\right)+t f^{\prime}\left(q^{0}\right) q^{1}+t^{2}\left\{f^{\prime}\left(q^{0}\right) q^{2}+\frac{1}{2} f^{\prime \prime}\left(q^{0}\right)\left(q^{1}\right)^{2}\right\}+\mathcal{O}\left(t^{3}\right) \quad$ for $t \rightarrow 0$,

and we see that $f^{1}\left(q^{0}\right)=0$ and $f^{2}\left(q^{0}, q^{1}\right)=\frac{1}{2} f^{\prime \prime}\left(q^{0}\right)\left(q^{1}\right)^{2}$. For a $2 \times 2$ system, we have

$$
\begin{aligned}
\left.\frac{\partial^{2} f_{p}(u)}{\partial t^{2}}\right|_{t=0}=\left(\frac{\partial^{2} f_{p}\left(q^{0}\right)}{\partial v^{2}}\left(v^{1}\right)^{2}\right. & +2 \frac{\partial f_{p}\left(q^{0}\right)}{\partial v} v^{2}+2 \frac{\partial^{2} f_{p}\left(q^{0}\right)}{\partial v \partial w} v^{1} w^{1} \\
& \left.+\frac{\partial^{2} f_{p}\left(q^{0}\right)}{\partial w^{2}}\left(w^{1}\right)^{2}+2 \frac{\partial f_{p}\left(q^{0}\right)}{\partial w} w^{2}\right)
\end{aligned}
$$

for $p=1,2$, and thus

$$
f(u(x, t))=f\left(q^{0}\right)+t A\left(q^{0}\right) q^{1}+t^{2}\left\{A\left(q^{0}\right) q^{2}+f^{2}\left(q^{0}, q^{1}\right)\right\}+\mathcal{O}\left(t^{3}\right) \quad \text { for } t \rightarrow 0,
$$

where

$$
f^{2}\left(q^{0}, q^{1}\right)=\frac{1}{2}\left(\frac{\partial^{2} f_{p}}{\partial v^{2}}\left(q^{0}\right)\left(v^{1}\right)^{2}+2 \frac{\partial^{2} f_{p}}{\partial v \partial w}\left(q^{0}\right) v^{1} w^{1}+\frac{\partial^{2} f_{p}}{\partial w^{2}}\left(q^{0}\right)\left(w^{1}\right)^{2}\right)_{p=1,2} .
$$

By that Taylor expansion of $f$ in powers of $t$ it is easy to see that $f^{k}$ is a polynomial of degree at most $k$, if every $q^{\ell}$ is a polynomial (in $\xi$ ) of degree at most $\ell$, for all $0 \leq \ell \leq k-1$.

Next, we combine (5.3) and (5.4) to find

$$
-\xi \frac{d q^{0}}{d \xi}+\frac{d}{d \xi} f\left(q^{0}\right)+\sum_{k \geq 1} t^{k}\left(k q^{k}-\xi \frac{d q^{k}}{d \xi}+\frac{d}{d \xi}\left(A\left(q^{0}\right) q^{k}+f^{k}\right)\right)=0,
$$

which yields for $k=0$,

$$
-\xi \frac{d q^{0}}{d \xi}+\frac{d}{d \xi} f\left(q^{0}\right)=0
$$

and for $k \geq 1$,

$$
k q^{k}-\xi \frac{d q^{k}}{d \xi}+\frac{d}{d \xi}\left(A\left(q^{0}\right) q^{k}+f^{k}\right)=0 .
$$

Letting $h^{k}(\xi)=-\frac{d}{d \xi} f^{k}\left(q^{0}, \ldots, q^{k-1}\right)$, this becomes

$$
k q^{k}-\xi \frac{d q^{k}}{d \xi}+\frac{d}{d \xi}\left(A\left(q^{0}\right) q^{k}\right)=h^{k} .
$$


We remark that in (5.6) the coefficient $A\left(q^{0}\right)$ depends on $q^{0}$ but not on $q^{k}$. Thus, (5.6) is a semi-linear equation. Moreover, recall that $f^{k}$ is a polynomial in $\xi$ of degree at most $k$, so $h^{k}$ is a polynomial of degree at most $k-1$.

5.3. Step II: Jump conditions. The above construction is valid wherever $u$ is smooth. So next we need to investigate the jump conditions satisfied by $q^{k}$ at the boundaries of the domains of smoothness of $u$. Take a curve $x=\gamma(t)$ that separates two domains of smoothness of $u$. Since these curves are all smooth, we can use a Taylor expansion to write

$$
\gamma(t)=\sigma^{0} t+\sigma^{1} t^{2}+\cdots+\sigma^{k-1} t^{k}+\ldots
$$

In fact, the solution $u$ is smooth, not only in $D_{i}$, but also in the closure $\bar{D}_{i}$; see [22]. So we can use, again, a Taylor expansion in powers of $t$ around the origin to obtain from (5.1) that

$$
\begin{aligned}
& u(\gamma(t), t)=\sum_{k \geq 0} t^{k} q^{k}\left(\frac{\gamma(t)}{t}\right)=\sum_{k \geq 0} t^{k} q^{k}\left(\sum_{\ell \geq 0} t^{\ell} \sigma^{\ell}\right) \\
& =q^{0}\left(\sigma^{0}\right)+t\left\{q^{1}\left(\sigma^{0}\right)+\sigma^{1} \frac{d q^{0}}{d \xi}\left(\sigma^{0}\right)\right\} \\
& +t^{2}\left\{q^{2}\left(\sigma^{0}\right)+\sigma^{2} \frac{d q^{0}}{d \xi}\left(\sigma^{0}\right)+z^{2}\left(\Sigma^{1}, Q^{1}\right)\right\}+\ldots \\
& +t^{k}\left\{q^{k}\left(\sigma^{0}\right)+\sigma^{k} \frac{d q^{0}}{d \xi}\left(\sigma^{0}\right)+z^{k}\left(\Sigma^{k-1}, Q^{k-1}\right)\right\}+\ldots,
\end{aligned}
$$

where the functions $z^{k}$ depend only on $\Sigma^{k-1}=\left(\sigma^{0}, \ldots, \sigma^{k-1}\right)$ and $Q^{k-1}=\left(q^{0}, \ldots\right.$, $\left.q^{k-1}\right)$. Similar to the $f^{k}$ in (5.4), we plug all terms belonging to $t^{k}$ that do not depend on $\sigma^{k}$ or $q^{k}$ in a Taylor expansion into this $z^{k}$. In particular, $z^{1}=0$ and

$$
z^{2}\left(\Sigma^{1}, Q^{1}\right)=\frac{1}{2}\left(\sigma^{1}\right)^{2} \frac{d^{2} q^{0}}{d \xi^{2}}\left(\sigma^{0}\right)+\sigma^{1} \frac{d q^{1}}{d \xi}\left(\sigma^{0}\right) .
$$

We denote the jump of a function $u$ at a point $\xi_{0}$ by

$$
[[u]]\left(\xi_{0}\right)=u\left(\xi_{0}+\right)-u\left(\xi_{0}-\right) .
$$

Therefore, if $u$ is continuous across the curve $x=\gamma(t)$, we simply get

$$
\left[\left[q^{0}\right]\right]\left(\sigma^{0}\right)=0
$$

from (5.7) for $k=0$. Moreover, for $k \geq 1$ we get

$$
\left[\left[q^{k}+\sigma^{k} \frac{d q^{0}}{d \xi}+z^{k}\left(\Sigma^{k-1}, Q^{k-1}\right)\right]\right]\left(\sigma^{0}\right)=0 .
$$

We see that $q^{0}$ is continuous at the point $\sigma^{0}$, whereas $q^{k}$ is in general discontinuous at $\sigma^{0}$ for $k \geq 1$.

Now let $u$ have a jump across the curve $x=\gamma(t)$. Then, by the Rankine-Hugoniot conditions,

$$
\dot{\gamma}(t)[[u]](x)=[[f(u)]](x), \quad x=\gamma(t) .
$$

To derive the correct jump conditions satisfied by the functions $q^{k}$, we will take the expansions for $f(u)$ and $\dot{\gamma} u$ along $x=\gamma(t)$, respectively. We start with the flux 
APPROXIMATE SOLUTIONS OF GENERALIZED RIEMANN PROBLEMS

45

along that curve of discontinuity: By a Taylor expansion around $t=0$ we get

$$
\begin{aligned}
& f(u(\gamma(t), t)) \\
& =f\left(\sum_{k \geq 0} t^{k}\left\{q^{k}\left(\sigma^{0}\right)+\sigma^{k} \frac{d}{d q^{0} \xi}\left(\sigma^{0}\right)+z^{k}\left(\Sigma^{k-1}, Q^{k-1}\right)\right\}\right) \\
& =f\left(q^{0}\left(\sigma^{0}\right)\right)+t A\left(q^{0}\left(\sigma^{0}\right)\right)\left(q^{1}\left(\sigma^{0}\right)+\sigma^{1} \frac{d q^{0}}{d \xi}\left(\sigma^{0}\right)\right) \\
& \quad+t^{2}\left\{A\left(q^{0}\left(\sigma^{0}\right)\right)\left(q^{2}\left(\sigma^{0}\right)+\sigma^{2} \frac{d q^{0}}{d \xi}\left(\sigma^{0}\right)\right)+a^{2}\left(\Sigma^{1}, Q^{1}\right)\right\}+\ldots \\
& \quad+t^{k}\left\{A\left(q^{0}\left(\sigma^{0}\right)\right)\left(q^{k}\left(\sigma^{0}\right)+\sigma^{k} \frac{d q^{0}}{d \xi}\left(\sigma^{0}\right)\right)+a^{k}\left(\Sigma^{k-1}, Q^{k-1}\right)\right\}+\ldots,
\end{aligned}
$$

where for the $2 \times 2$ system we can express $a^{2}$ explicitly as $a^{2}\left(\Sigma^{1}, Q^{1}\right)$

$$
\begin{aligned}
= & \frac{1}{2}\left(\frac{\partial f_{p}}{\partial v}\left(q^{0}\left(\sigma^{0}\right)\right)\left\{\left(\sigma^{1}\right)^{2} \frac{d^{2} v^{0}}{d \xi^{2}}\left(\sigma^{0}\right)+\sigma^{1} \frac{d v^{1}}{d \xi}\left(\sigma^{0}\right)\right\}\right. \\
& +\frac{\partial f_{p}}{\partial w}\left(q^{0}\left(\sigma^{0}\right)\right)\left\{\left(\sigma^{1}\right)^{2} \frac{d^{2} w^{0}}{d \xi^{2}}\left(\sigma^{0}\right)+\sigma^{1} \frac{d w^{1}}{d \xi}\left(\sigma^{0}\right)\right\} \\
& +\frac{\partial^{2} f_{p}}{\partial v^{2}}\left(q^{0}\left(\sigma^{0}\right)\right)\left\{v^{1}\left(\sigma^{0}\right)+\sigma^{1} \frac{d v^{0}}{d \xi}\left(\sigma^{0}\right)\right\}^{2} \\
& +\frac{\partial^{2} f_{p}}{\partial w^{2}}\left(q^{0}\left(\sigma^{0}\right)\right)\left\{w^{1}\left(\sigma^{0}\right)+\sigma^{1} \frac{d w^{0}}{d \xi}\left(\sigma^{0}\right)\right\}^{2} \\
& \left.+2 \frac{\partial^{2} f_{p}}{\partial v \partial w}\left(q^{0}\left(\sigma^{0}\right)\right)\left\{v^{1}\left(\sigma^{0}\right)+\sigma^{1} \frac{d v^{0}}{d \xi}\left(\sigma^{0}\right)\right\}\left\{w^{1}\left(\sigma^{0}\right)+\sigma^{1} \frac{d w^{0}}{d \xi}\left(\sigma^{0}\right)\right\}\right)_{p=1,2} .
\end{aligned}
$$

Furthermore, we have

$$
\begin{aligned}
\dot{\gamma}(t) u(\gamma(t), t) & \\
= & \sigma^{0} q^{0}\left(\sigma^{0}\right)+t\left\{\sigma^{0}\left(q^{1}\left(\sigma^{0}\right)+\sigma^{1} \frac{d q^{0}}{d \xi}\left(\sigma^{0}\right)\right)+2 \sigma^{1} q^{0}\left(\sigma^{0}\right)\right\} \\
& +t^{2}\left\{\sigma^{0}\left(q^{2}\left(\sigma^{0}\right)+\sigma^{2} \frac{d q^{0}}{d \xi}\left(\sigma^{0}\right)\right)+3 \sigma^{2} q^{0}\left(\sigma^{0}\right)+b^{2}\left(\Sigma^{1}, Q^{1}\right)\right\}+\ldots \\
& +t^{k}\left\{\sigma^{0}\left(q^{k}\left(\sigma^{0}\right)+\sigma^{k} \frac{d q^{0}}{d \xi}\left(\sigma^{0}\right)\right)+(k+1) \sigma^{k} q^{0}\left(\sigma^{0}\right)+b^{k}\left(\Sigma^{k-1}, Q^{k-1}\right)\right\}+\ldots
\end{aligned}
$$

with

$$
b^{2}\left(\Sigma^{1}, Q^{1}\right)=2 \sigma^{1}\left(q^{1}\left(\sigma^{0}\right)+\sigma^{1} \frac{d q^{0}}{d \xi}\left(\sigma^{0}\right)\right)+\sigma^{0} z^{2}\left(\Sigma^{1}, Q^{1}\right) .
$$

In summary, at $\xi=\sigma^{0}$ the jump conditions are

$$
\sigma^{0}\left[\left[q^{0}\right]\right]=\left[\left[f\left(q^{0}\right)\right]\right] \text { at } \sigma^{0},
$$

for $k=0$, and for $k \geq 1$ we get $(5.14)$

$$
\left[\left[\left(A\left(q^{0}\right)-\sigma^{0}\right) q^{k}\right]\right]+\sigma^{k}\left[\left[\left(A\left(q^{0}\right)-\sigma^{0}\right) \frac{d q^{0}}{d \xi}\right]\right]-\sigma^{k}\left[\left[(k+1) q^{0}\right]\right]+\left[\left[c^{k}\right]\right]=0 \text { at } \sigma^{0}
$$


with a function $c^{k}\left(\Sigma^{k-1}, Q^{k-1}\right)=a^{k}\left(\Sigma^{k-1}, Q^{k-1}\right)-b^{k}\left(\Sigma^{k-1}, Q^{k-1}\right)$.

Finally, we remark that for $|\xi|$ large enough, say $|\xi| \geq \xi_{0}$,

$$
q^{0}(\xi)= \begin{cases}\hat{u}_{R}^{0}, & \xi>\xi_{0}, \\ \hat{u}_{L}^{0}, & \xi<-\xi_{0} .\end{cases}
$$

We now can summarize the above construction:

Lemma 5.1. The function $q^{0}$ satisfies the relations (5.5), (5.9), (5.13), (5.15), which characterize the piecewise continuous self-similar entropy solution $q^{0}(x, t)=$ $q^{0}(\xi)$ of the Riemann problem

$$
\begin{aligned}
& \frac{\partial}{\partial t} q^{0}(x, t)+\frac{\partial}{\partial x} f\left(q^{0}(x, t)\right)=0, \\
& q^{0}(x, 0)= \begin{cases}\hat{u}_{L}^{0}, & \text { if } x<0, \\
\hat{u}_{R}^{0}, & \text { if } x>0 .\end{cases}
\end{aligned}
$$

Therefore, we can conclude that the Toro-Titarev solver sets up "the right problem", when it comes to computing the leading term of the expansion.

5.4. Step III: Higher order terms. Assume that the solution of (5.16), (5.17) contains no transonic rarefaction wave. Then line segment $\{x=0\} \times[0, \Delta t]$ is contained in a domain of smoothness, say in $D_{i *}$. Since we do not explicitly need the expansion inside the rarefaction zones, we only consider the simplified case that the solution $q^{0}$ of contains only shock waves or contact discontinuities. The full problem requires similar techniques, although some of the details are more involved (again, see 20] for the full construction). When we only have shocks and contact discontinuities, the solution $q^{0}$ of (5.16), (5.17) has the form

$$
q^{0}(\xi)= \begin{cases}q_{0}^{0}=\hat{u}_{L}^{0}, & \text { for } \xi \in\left(-\infty, \sigma_{1}^{0}\right), \\ q_{i}^{0}, & \text { for } \xi \in\left(\sigma_{i}^{0}, \sigma_{i+1}^{0}\right), 1 \leq i \leq m-1, \\ q_{m}^{0}=\hat{u}_{R}^{0}, & \text { for } \xi \in\left(\sigma_{m}^{0}, \infty\right) .\end{cases}
$$

Now consider the domains in which $q^{0}$ takes the constant value $q_{i}^{0}$,

$$
D_{i}^{0}=\left\{(x, t) \mid \sigma_{i}^{0}<\xi<\sigma_{i+1}^{0}\right\}, \quad i=0, \ldots, m .
$$

As a convention, we let $\sigma_{0}^{0}=-\infty, \sigma_{m+1}^{0}=+\infty$. Then equation (5.6) in $D_{i}^{0}$ becomes

$$
k q^{k}+\left(A\left(q_{i}^{0}\right)-\xi\right) \frac{d}{d \xi} q^{k}=h^{k} .
$$

Recall that $h^{k}$ is a polynomial of degree at most $k-1$. It is then straightforward to show that the general solution of (5.18) is given by

$$
q^{k}(\xi)=\left(\xi-A\left(q_{i}^{0}\right)\right)^{k} q_{i}^{k}+p_{i}^{k}(\xi),
$$

where $q_{i}^{k} \in \mathbb{R}^{m}$ is an arbitrary vector and $p_{i}^{k}: \mathbb{R} \rightarrow \mathbb{R}^{m}$ is a polynomial of degree at most $k-1$ with coefficients that depend only on $q^{0}, \ldots, q^{k-1}$.

More precisely, $\left(\xi-A\left(q_{i}^{0}\right)^{k} q_{i}^{k}\right.$ is a solution of the homogeneous part of (5.18) and $p_{i}^{k}$ is a particular solution of (5.18). Since $f^{1}=0$, we have $h^{1}=0$, and therefore, $p^{1}=0$. For the $2 \times 2$ system, this means that

$$
\left(\begin{array}{c}
v^{1}(\xi) \\
w^{1}(\xi)
\end{array}\right)=\left(\begin{array}{l}
\xi q_{i, 1}^{1}-\frac{\partial f_{1}}{\partial v}\left(q_{i}^{0}\right) q_{i, 1}^{1}-\frac{\partial f_{1}}{\partial w}\left(q_{i}^{0}\right) q_{i, 2}^{1} \\
\xi q_{i, 2}^{1}-\frac{\partial f_{2}}{\partial v}\left(q_{i}^{0}\right) q_{i, 1}^{1}-\frac{\partial f_{2}}{\partial w}\left(q_{i}^{0}\right) q_{1,2}^{1}
\end{array}\right)
$$


where we denote $q_{i}^{1}=\left(q_{i, 1}^{1}, q_{i, 2}^{1}\right)^{T}$. Then we get

$$
\begin{aligned}
h^{2}(\xi)= & -v^{1}(\xi)\left(\frac{\partial^{2} f_{p}}{\partial v^{2}}\left(q_{1}^{0}\right) q_{1,1}^{1}+\frac{\partial^{2} f_{p}}{\partial v \partial w}\left(q_{1}^{0}\right) q_{1,2}^{1}\right)_{p=1,2} \\
& -w^{1}(\xi)\left(\frac{\partial^{2} f_{p}}{\partial v \partial w}\left(q_{1}^{0}\right) q_{1,1}^{1}+\frac{\partial^{2} f_{p}}{\partial w^{2}}\left(q_{1}^{0}\right) q_{1,2}^{1}\right)_{p=1,2} .
\end{aligned}
$$

In general, writing

$$
h_{i}^{k}(\xi)=\sum_{\ell=0}^{k-1} \beta_{i}^{\ell} \xi^{\ell} \quad \text { and } \quad p_{i}^{k}(\xi)=\sum_{\ell=0}^{k-1} \theta_{i}^{\ell} \xi^{\ell},
$$

the coefficients $\theta_{i}^{\ell}$ of the polynomial $p_{i}^{k}$ can be obtained as follows (cf. [20, Lemma 2]).

$$
\begin{aligned}
\theta_{i}^{k-1} & =\beta_{i}^{k-1} \\
(\ell+1) A\left(q_{i}^{0}\right) \theta_{i}^{\ell+1}+(k-\ell) \theta_{i}^{\ell} & =\beta_{i}^{\ell} \quad \text { for } 0 \leq \ell \leq k-2 .
\end{aligned}
$$

Recall that the function $q^{0}$ is piecewise constant. This allows us to simplify some of the above expressions. Let $u$ have a jump across the curve $x=\gamma_{i}(t)$, then we have

$$
q^{0}\left(\sigma_{i}^{0}-\right)=q_{i-1}^{0}, \quad q^{0}\left(\sigma_{i}^{0}+\right)=q_{i}^{0}, \quad \frac{d q^{0}}{d \xi}\left(\sigma_{i}^{0}-\right)=\frac{d q^{0}}{d \xi}\left(\sigma_{i}^{0}+\right)=0,
$$

and thus, using the representation (5.19), we get from (5.8) for the $2 \times 2$ system

$$
z^{2}\left(\Sigma^{1}, Q^{1}\right)=\sigma_{i}^{1} \frac{d q^{1}}{d \xi}\left(\sigma^{0}\right)=\sigma_{i}^{1} q_{i}^{1} .
$$

This gives $b^{2}\left(\Sigma^{1}, Q^{1}\right)=\sigma_{i}^{1}\left(2 q^{1}\left(\sigma_{i}^{0}\right)+\sigma_{i}^{0} q_{i}^{1}\right)$. Moreover (5.11), reduces to

$$
\begin{aligned}
a^{2}\left(\Sigma^{1}, Q^{1}\right)=\frac{1}{2}\left(\sigma^{1}\right. & \left\{\frac{\partial f_{p}}{\partial v}\left(q^{0}\left(\sigma^{0}\right)\right) \frac{d v^{1}}{d \xi}\left(\sigma^{0}\right)+\frac{\partial f_{p}}{\partial w}\left(q^{0}\left(\sigma^{0}\right) \frac{d w^{1}}{d \xi}\left(\sigma^{0}\right)\right\}\right. \\
& +\frac{\partial^{2} f_{p}}{\partial v^{2}}\left(q^{0}\left(\sigma^{0}\right)\right)\left(v^{1}\left(\sigma^{0}\right)\right)^{2}+\frac{\partial^{2} f_{p}}{\partial w^{2}}\left(q^{0}\left(\sigma^{0}\right)\right)\left(w^{1}\left(\sigma^{0}\right)\right)^{2} \\
& \left.+2 \frac{\partial^{2} f_{p}}{\partial v \partial w}\left(q^{0}\left(\sigma^{0}\right)\right) v^{1}\left(\sigma^{0}\right) w^{1}\left(\sigma^{0}\right)\right)_{p=1,2}
\end{aligned}
$$

\section{Connecting the Toro-Titarev solver with the LEFLOCH-RAVIART SOLVER}

Let us take a look at the Taylor expansion that we used to define the functions $q^{k}$ : We consider the domains

$$
D_{i}=\left\{\xi \mid \frac{\gamma_{i-1}(t)}{t}<\xi<\frac{\gamma_{i}(t)}{t}\right\}
$$

Since we have $\gamma_{i}(0)=0, \dot{\gamma}_{i}(0)=\sigma_{i}^{0}$, the domains remain close to the domains $D_{i}^{0}$ in which $u^{0}$ is constant, for small $t>0$. Inside each domain of smoothness $D_{i}$ we may take a Taylor expansion around some $\left(x_{0}, t_{0}\right) \in D_{i}$ and define the Taylor 
expansion at the origin by the limit $\left(x_{0}, t_{0}\right) \rightarrow(0,0+) \in D_{i}$. In that sense the Taylor expansion around the origin gives

$$
\begin{aligned}
u(x, t) & =\sum_{k=0}^{\infty} \sum_{\ell=0}^{k} \frac{\partial^{\ell}}{\partial x^{\ell}} \frac{\partial^{k-\ell}}{\partial t^{k-\ell}} \frac{u(0,0+)}{\ell !(k-\ell) !} x^{\ell} t^{k-\ell} \\
& =u(0,0+)+\sum_{k=1}^{\infty} t^{k} \sum_{\ell=0}^{k} \frac{\partial^{\ell}}{\partial x^{\ell}} \frac{\partial^{k-\ell}}{\partial t^{k-\ell}} \frac{u(0,0+)}{\ell !(k-\ell) !}\left(\frac{x}{t}\right)^{\ell} .
\end{aligned}
$$

Thus, the vector $q_{i}^{k}$ in (5.19), which gives the leading coefficient of this polynomial, defines the value $\frac{\partial^{k} u}{\partial x^{k}}(0,0+)$.

To determine the vectors $q_{i}^{k}$, we first describe $q_{0}^{k}$ and $q_{m}^{k}$, as in [20, Lemma 6]. Using the notation from before, for the initial data we can write

$$
\hat{u}_{L}(x)=\hat{u}_{L}^{0}+\sum_{k=1}^{r-1} \frac{\hat{u}_{L}^{k}}{k !} x^{k}, \quad \hat{u}_{R}(x)=\hat{u}_{R}^{0}+\sum_{k=1}^{r-1} \frac{\hat{u}_{R}^{k}}{k !} x^{k} .
$$

In $D_{0}$, the solution is given by the functions

$$
q^{k}(\xi)=\left(\xi-A\left(q_{0}^{0}\right)\right)^{k} q_{0}^{k}+p_{0}^{k}(\xi) .
$$

Since $p_{0}^{k}$ is a polynomial of degree at most $k-1$, we find

$$
\lim _{\substack{t \rightarrow 0 \\ x<\gamma_{1}(t)}} t^{k} q^{k}\left(\frac{x}{t}\right)=x^{k} q_{0}^{k} .
$$

Hence, it follows that

$$
u(x, 0)=\lim _{\substack{t \rightarrow 0 \\ x<\gamma_{1}(t)}} u(x, t)=q_{0}^{0}+\sum_{k=1}^{r-1} q_{0}^{k} x^{k} .
$$

Therefore, we have

$$
q_{0}^{k}=\frac{\hat{u}_{L}^{k}}{k !}, \quad \text { for } k=0, \ldots, r-1 .
$$

Analogously, we get $q_{m}^{k}=\hat{u}_{R}^{k} / k !, k=0, \ldots, r-1$.

Now consider the scalar case. For a strictly convex flux, $f^{\prime \prime}>0$, we only have two domains of smoothness. In that case, all coefficients $q_{i}^{k}, i=0,1$, and $k=0, \ldots, r-1$ are uniquely determined by the initial data and its derivatives. Assuming that there is no transonic wave, solving linear RPs merely means picking the left or the right side, depending on the sign of the coefficient in the evolution equation. Thus, to build the expansion, we first have to solve one nonlinear RP to determine which domain of smoothness contains the line segment $\{x=0\} \times[0, \Delta t]$. Then, use the data from that side, which is equivalent to solving linear RPs. So the solver of Toro and Titarev reproduces the first $r-1$ terms of the LeFloch-Raviart solver exactly. In summary, we can state one main result of this paper.

Theorem 6.1. Consider the generalized Riemann problem for a scalar, nonlinear hyperbolic conservation law in one spatial dimension with strictly convex flux. Let the initial data consist of piecewise polynomials of degree $r-1$. Assume the solution 
does not contain a transonic wave. Then, the Toro-Titarev solver and the LeFlochRaviart expansion yield the same truncated Taylor expansion in time at $x=0$,

$$
\sum_{k=0}^{r-1} \Phi^{k}\left(u^{0}, \ldots, u^{k}\right)(0) \frac{\tau^{k}}{k !}=\sum_{k=0}^{r-1} q^{k}(0) \tau^{k}=\mathcal{E}(\tau) \hat{u}(0)+\mathcal{O}\left(\Delta t^{r}\right)
$$

for $0<\tau<\Delta t$ as $\Delta t \rightarrow 0+$.

Indeed, both methods are computing the same truncated Taylor expansion. The difference is, however, that the Toro-Titarev solver uses an approximation to the spatial derivatives at the origin. For illustration, we check that for a scalar problem both methods formally construct the expansion up to quadratic terms. Assume that $\sigma_{i}^{0}<0<\sigma_{i+1}^{0}$ and consider the Taylor approximation inside $D_{i}$,

$$
\begin{aligned}
u(x, t) & \approx u(0,0+)+t\left\{\frac{\partial u(0,0+)}{\partial x} \frac{x}{t}+\frac{\partial u(0,0+)}{\partial t}\right\} \\
& +t^{2}\left\{\frac{1}{2} \frac{\partial^{2} u(0,0+)}{\partial x^{2}}\left(\frac{x}{t}\right)^{2}+\frac{\partial^{2} u(0,0+)}{\partial x \partial t}\left(\frac{x}{t}\right)+\frac{1}{2} \frac{\partial^{2} u(0,0+)}{\partial t^{2}}\right\},
\end{aligned}
$$

where function evaluations and derivatives at $(0,0+)$ are regarded as limits $D_{i} \ni$ $(x, t) \rightarrow(0,0+)$. The Cauchy-Kowalewskaya procedure now gives:

$$
\begin{aligned}
\frac{\partial u}{\partial t} & =-f^{\prime}(u) \frac{\partial u}{\partial x}, \quad \frac{\partial^{2} u}{\partial x \partial t}=-f^{\prime \prime}(u)\left(\frac{\partial u}{\partial x}\right)^{2}-f^{\prime}(u) \frac{\partial^{2} u}{\partial x^{2}}, \\
\frac{\partial^{2} u}{\partial t^{2}} & =2 f^{\prime}(u) f^{\prime \prime}(u)\left(\frac{\partial u}{\partial x}\right)^{2}+\left(f^{\prime}(u)\right)^{2} \frac{\partial^{2} u}{\partial x^{2}} .
\end{aligned}
$$

Inserting this into the above Taylor approximation yields

$$
\begin{aligned}
& u(x, t) \approx u(0,0+)+t\left\{\left(\frac{x}{t}-f^{\prime}(u(0,0+))\right) \frac{\partial u}{\partial x}(0,0+)\right\} \\
& +t^{2}\left\{\left(\frac{x}{t}-f^{\prime}(u(0,0+))\right)^{2} \frac{1}{2} \frac{\partial^{2} u}{\partial x^{2}}(0,0+)-f^{\prime \prime}(u(0,0+))\left(\frac{\partial u}{\partial x}(0,0+)\right)^{2}\left(\frac{x}{t}\right)\right. \\
& \left.+f^{\prime}(u(0,0+)) f^{\prime \prime}(u(0,0+))\left(\frac{\partial u}{\partial x}(0,0+)\right)^{2}\right\} .
\end{aligned}
$$

Now let us compute us the terms up to $q^{2}$ in the LeFloch-Raviart solver. We have

$$
q_{i}^{1}(\xi)=\left(\xi-f^{\prime}\left(q_{i}^{0}\right)\right) q_{i}^{1} .
$$

For $q^{2}$, we first compute

$$
\begin{aligned}
h^{2}(\xi)=-\frac{d}{d \xi} f^{2}\left(q_{i}^{0}(\xi), q_{i}^{1}(\xi)\right) & =-\frac{1}{2} \frac{d}{d \xi}\left(f^{\prime \prime}\left(q_{i}^{0}\right)\left(q_{i}^{1}(\xi)\right)^{2}\right) \\
& =-f^{\prime \prime}\left(q_{i}^{0}\right)\left(\xi-f^{\prime}\left(q_{i}^{0}\right)\right)\left(q_{i}^{0}\right)^{2}=\beta_{i}^{1} \xi+\beta_{i}^{0},
\end{aligned}
$$

where $\beta_{i}^{1}=-f^{\prime \prime}\left(q_{i}^{0}\right)\left(q_{i}^{1}\right)^{2}$ and $\beta_{i}^{0}=f^{\prime}\left(q_{i}^{0}\right) f^{\prime \prime}\left(q_{i}^{0}\right)\left(q_{i}^{1}\right)^{2}$. By letting $p_{i}^{2}(\xi)=\theta_{i}^{1} \xi+\theta_{i}^{0}$, we get

$$
\theta_{i}^{1}=\beta_{i}^{1}, \quad \theta_{i}^{0}=\frac{1}{2}\left(\beta_{i}^{0}-f^{\prime}\left(q_{i}^{0}\right) \theta_{i}^{1}\right)=f^{\prime}\left(q_{i}^{0}\right) f^{\prime \prime}\left(q_{i}^{0}\right)\left(q_{i}^{1}\right)^{2} .
$$

Thus, we have

$$
q_{i}^{2}(\xi)=\left(\xi-f^{\prime}\left(q_{i}^{0}\right)\right)^{2} q_{i}^{2}-f^{\prime \prime}\left(q_{i}^{0}\right)\left(q_{i}^{1}\right)^{2} \xi+f^{\prime}\left(q_{i}^{0}\right) f^{\prime \prime}\left(q_{i}^{0}\right)\left(q_{i}^{1}\right)^{2} .
$$


Noting that $q_{i}^{k}=\frac{1}{k !} \frac{\partial^{k} u}{\partial x^{k}}(0,0+)$, we see that $q^{0}+t q^{1}+t^{2} q^{2}$ agrees with (6.1).

Naturally, the question arises whether this result can be extended to the case of systems. What we compare are the coefficient $q_{i *}^{k}$ and the Godunov state for the $k$-th spatial derivative in the Toro-Titarev solver. To do so, at first we write each coefficient $q_{i}^{k}$ in the form

$$
q_{i}^{k}=\sum_{j=1}^{m} \alpha_{i j}^{k} r_{j}\left(q_{i}^{0}\right) .
$$

Note that the coefficients $\alpha_{0, j}^{k}, \alpha_{m, j}^{k}, j=1, \ldots, m$ are known from the initial data. To characterize the coefficients $\alpha_{i j}^{k}, i=1, \ldots, m-1$, we need the following two results.

Lemma 6.2 (cf. [20, Lemma 4]). Assume that the $i$-th wave is a shock wave or a contact discontinuity. Then for all $k \geq 1$, there exists a vector $s_{i}^{k} \in \mathbb{R}^{m}$, depending only on $q^{0}, \ldots, q^{k}, \sigma_{i}^{0}, \ldots, \sigma_{i}^{k-1}$ such that

$$
\left(A\left(q_{i}^{0}\right)-\sigma_{i}^{0}\right)^{k+1} q_{i}^{k}=\left(A\left(q_{i-1}^{0}\right)-\sigma_{i}^{0}\right)^{k+1} q_{i-1}^{k}+(-1)^{k}(k+1) \sigma_{i}^{k}\left(q_{i}^{0}-q_{i-1}^{0}\right)+s_{i}^{k} .
$$

More precisely, (6.2) holds with

$s_{i}^{k}=(-1)^{k+1}\left(\left(A\left(q_{i}^{0}\right)-\sigma_{i}^{0}\right) p_{i}^{k}\left(\sigma_{i}^{0}\right)-\left(A\left(q_{i-1}^{0}\right)-\sigma_{i}^{0}\right) p_{i-1}^{k}\left(\sigma_{i}^{0}\right)\right)+c_{i}^{k}\left(\sigma_{i}^{0}+\right)-c_{i}^{k}\left(\sigma_{i}^{0}-\right)$.

Lemma 6.3 (Corollary from Theorem 1 in 20]). Assume the $i$-th wave is a shock wave or a contact discontinuity. Then, we have for $i \neq j$ :

$$
\begin{array}{r}
\sum_{p=1}^{m}\left(\lambda_{p}\left(q_{i}^{0}\right)-\sigma_{i}^{0}\right)^{k+1} \ell_{j}\left(q_{i-1}^{0}\right) \cdot r_{p}\left(q_{i}^{0}\right) \alpha_{i p}^{k}-\left(\lambda_{j}\left(q_{i-1}^{0}\right)-\sigma_{i}^{0}\right)^{k+1} \alpha_{i-1, j}^{k} \\
=\frac{\ell_{j}\left(q_{i-1}^{0}\right) \cdot\left(q_{i}^{0}-q_{i-1}^{0}\right)}{l_{i}\left(q_{i-1}^{0}\right) \cdot\left(q_{i}^{0}-q_{i-1}^{0}\right)}\left\{\sum_{p=1}^{m}\left(\lambda_{p}\left(q_{i}^{0}\right)-\sigma_{i}^{0}\right)^{k+1} \ell_{i}\left(q_{i-1}^{0}\right) \cdot r_{p}\left(q_{i}^{0}\right) \alpha_{i p}^{k}\right. \\
\left.\quad-\left(\lambda_{i}\left(q_{i-1}^{0}\right)-\sigma_{i}^{0}\right)^{k+1} \alpha_{i-1, i}^{k}-\ell_{i}\left(q_{i-1}^{0}\right) \cdot s_{i}^{k}\right\}+\ell_{j}\left(q_{i-1}^{0}\right) \cdot s_{i}^{k} .
\end{array}
$$

Both statements are derived from the jump relation (5.14). LeFloch and Raviart show that this leads to a uniquely solvable system of linear equations for the coefficients $\alpha_{i j}^{k}$ (cf. [20, Theorem 1]). The question of whether this gives coefficients $q_{i}^{k}$ that agree with the intermediate states in the linear RPs of the Toro-Titarev solver is answered by the following theorem, which is the other main result of this paper.

Theorem 6.4. Consider the generalized Riemann problem for a strictly hyperbolic $m \times m$ system of conservation laws, such that every characteristic field is either genuinely nonlinear or linearly degenerate. Let the initial data consist of polynomials $\hat{u}_{L}, \hat{u}_{R}$ of degree $r-1$ with sufficiently small $\left|\hat{u}_{L}(0)-\hat{u}_{R}(0)\right|>0$. Assume that the solution contains only shock waves and contact discontinuities. Then, for $k \geq 1$ the coefficients $q_{i}^{k}$ in the LeFloch-Raviart expansion and the states $u_{i}^{k}$ in the linear Riemann problems of the Toro-Titarev solver satisfy the relation

$$
q_{i}^{k}=\frac{1}{k !} u_{i}^{k} \quad \text { for } i=0 \text { and for } i=m .
$$

This does, in general, not hold for $1 \leq i \leq m-1$. 
Proof. The statement that

$$
q_{0}^{k}=\frac{1}{k !} u_{0}^{k}, \quad q_{m}^{k}=\frac{1}{k !} u_{m}^{k}, \quad k=1, \ldots, r-1,
$$

was already shown in our discussion of the scalar case. Now take (5.14) for $k=1$, in which case we have $c^{1}=0$ and $s_{i}^{1}=0$, so (5.14) becomes

$$
\left[\left[\left(A\left(q^{0}\right)-\sigma_{i}^{0}\right) q^{1}\right]\right]-\sigma_{i}^{1}\left[\left[\left(A\left(q^{0}\right)-\sigma_{i}^{0}\right) \frac{d}{d \xi} q^{0}-2 q^{0}\right]\right]=0 \quad \text { at } \sigma_{i}^{0} .
$$

We note that

$$
q^{0}\left(\sigma_{i}^{0}-0\right)=q_{i-1}^{0}, \quad q^{0}\left(\sigma_{i}^{0}+0\right)=q_{i}^{0}, \quad \frac{d}{d \xi} q^{0}\left(\sigma_{i}^{0} \pm 0\right)=0,
$$

and thus, using the solution formula (5.19) to represent $q^{1}$, the jump condition (6.4) becomes

$$
\left(A\left(q_{i}^{0}\right)-\sigma_{i}^{0}\right)^{2} q_{i}^{1}-\left(A\left(q_{i-1}^{0}\right)-\sigma_{i}^{0}\right)^{2} q_{i-1}^{1}-2 \sigma_{i}^{1}\left(q_{i}^{0}-q_{i-1}^{0}\right)=0 .
$$

Now consider the Toro-Titarev solver. We denote the solution of the linearised RP (4.5) by $u^{k}$ and let $u_{i}^{k}, i=0, \ldots, n$ be the constant states in that solution. If $u_{i *}^{0}$ is the Godunov state for $u^{0}$, then solving the RPs linearised around $u_{i *}^{0}$ is equivalent to imposing the jump conditions

$$
\left(A\left(u_{i *}^{0}\right)-\lambda_{i}\left(u_{i *}^{0}\right)\right)\left(u_{i}^{1}-u_{i-1}^{1}\right)=0, \quad i=1, \ldots, m .
$$

Clearly, (6.5) and (6.6) do not have the same solution.

We remark, however, that when all states $q_{i}^{0}$ are close, the solutions of (6.5) and (6.6) are close. This depends only on the leading term $q^{0}$, but not on higher order terms. Thus, when the jump in the initial data $\left|\hat{u}_{L}^{0}-\hat{u}_{R}^{0}\right|$ is small we expect (6.6) to give a good approximation to (6.5).

\section{ApPlications AND NUMERICAL EXAMPles}

7.1. Two-component chromatography. Consider the system

$$
\frac{\partial}{\partial t}\left(\begin{array}{c}
v \\
w
\end{array}\right)+\frac{\partial}{\partial x}\left(\begin{array}{c}
v(1+v+w)^{-1} \\
w(1+v+w)^{-1}
\end{array}\right), \quad v, w>0
$$

and denote $u=(v, w)^{T}, u \in \mathcal{U}=(0, \infty) \times(0, \infty) \subset \mathbb{R}^{2}$. This example is inspired by the analysis of two-component chromatography, as described by Temple 28. A discussion on the RP for (7.1) can be found in [7]. The Jacobian of the flux is given as

$$
A(v, w)=\frac{1}{(1+v+w)^{2}}\left(\begin{array}{cc}
1+w & -v \\
-w & 1+v
\end{array}\right)
$$

with eigenvalues

$$
\lambda_{1}(u)=\frac{1}{(1+v+w)^{2}}, \quad \lambda_{2}(u)=\frac{1}{1+v+w} .
$$

The corresponding (normalized) right eigenvectors are

$$
r_{1}(u)=\frac{1}{\sqrt{v^{2}+w^{2}}}\left(\begin{array}{c}
-v \\
-w
\end{array}\right), \quad r_{2}(u)=\frac{1}{\sqrt{2}}\left(\begin{array}{c}
1 \\
-1
\end{array}\right),
$$


and the left eigenvectors, normalized to $\ell_{j}(v, w) \cdot r_{i}(v, w)=\delta_{i j}$, are

$$
\ell_{1}(v, w)=-\frac{\sqrt{v^{2}+w^{2}}}{v+w}\left(\begin{array}{l}
1 \\
1
\end{array}\right), \quad \ell_{2}(v, w)=-\frac{\sqrt{2}}{v+w}\left(\begin{array}{c}
-w \\
v
\end{array}\right) .
$$

The first characteristic field is genuinely nonlinear, while the second is linearly degenerate.

For this system, shock and rarefaction curves coincide in the sense that each point in the $i$-Hugoniot set $(i=1,2)$ of a given point $u_{-}$lies on the integral curve of $r_{i}$ through $u_{-}$. Due to the simple nature of the eigenvectors, the integral curves here are straight lines in the space of conserved variables.

Now consider the RP with initial data $u_{L}=\left(v_{L}, w_{L}\right)^{T}, u_{R}=\left(v_{R}, w_{R}\right)^{T}$. Then the Riemann solution contains the states $u_{0}=u_{L}, u_{1}=\left(v_{1}, w_{1}\right)^{T}$, and $u_{2}=u_{R}$, so that

$$
\begin{aligned}
& \left(\begin{array}{c}
v_{1} \\
w_{1}
\end{array}\right)=\left(\begin{array}{c}
v_{0} \\
w_{0}
\end{array}\right)+\frac{\varepsilon_{1}}{\sqrt{v_{0}^{2}+w_{0}^{2}}}\left(\begin{array}{c}
-v_{0} \\
-w_{0}
\end{array}\right), \\
& \left(\begin{array}{c}
v_{2} \\
w_{2}
\end{array}\right)=\left(\begin{array}{c}
v_{1} \\
w_{1}
\end{array}\right)+\frac{\varepsilon_{2}}{\sqrt{2}}\left(\begin{array}{c}
1 \\
-1
\end{array}\right),
\end{aligned}
$$

for some $\varepsilon_{1}, \varepsilon_{2}$. Since the second field is linearly degenerate, we have $\lambda_{2}\left(u_{1}\right)=$ $\lambda_{2}\left(u_{2}\right)$ and therefore

$$
v_{1}+w_{1}=v_{2}+w_{2} .
$$

Furthermore, it follows from (7.2) that

$$
v_{0} w_{1}=v_{1} w_{2} .
$$

Combing the conditions (7.4) and (7.5), we can explicitly compute

$$
v_{1}=\frac{v_{0}\left(v_{2}+w_{2}\right)}{v_{0}+w_{0}}, \quad w_{1}=\frac{w_{0}\left(v_{2}+w_{2}\right)}{v_{0}+w_{0}},
$$

and the wave strength $\varepsilon_{1}$ is

$$
\varepsilon_{1}=\left(1-\frac{v_{2}+w_{2}}{v_{0}+w_{0}}\right) \sqrt{v_{0}^{2}+w_{0}^{2}} .
$$

Recall that the type of wave associated with the first characteristic family depends on the sign of $\varepsilon_{1}$ : We get a 1-shock for $\varepsilon_{1} \leq 0$ and a 1-rarefaction for $\varepsilon_{1}>0$ (the second wave is always a contact discontinuity, independent of the sign of $\varepsilon_{2}$ ).

Thus, if $v_{2}+w_{2} \geq v_{0}+w_{0}$, the solution contains a 1-shock and a 2-contact discontinuity. The shock speed $\sigma_{1}$ can be computed from the Rankine-Hugoniot conditions:

$$
\sigma_{1}=\int_{0}^{1} \lambda_{1}\left(\theta u_{1}+(1-\theta) u_{0}\right) d \theta=\frac{1}{\left(1+v_{0}+w_{0}\right)\left(1+v_{1}+w_{1}\right)} .
$$

For the contact discontinuity we have $\sigma_{2}=\lambda_{2}\left(u_{1}\right)=\lambda_{2}\left(u_{2}\right)$.

Now consider the GRP with piecewise linear initial data,

$$
\hat{u}_{a}(x)=\left(\begin{array}{c}
\hat{v}_{a}(x) \\
\hat{w}_{a}(x)
\end{array}\right)=\left(\begin{array}{c}
\hat{v}_{a}^{0} \\
\hat{w}_{a}^{0}
\end{array}\right)+x\left(\begin{array}{c}
\hat{v}_{a}^{1} \\
\hat{w}_{a}^{1}
\end{array}\right),
$$

for $a=L, R$. Denote $\hat{u}_{a}^{k}=\left(v_{a}^{k}, w_{a}^{k}\right)^{T}$ for $a=L, R$, and $k=0,1$ and let $u^{0}$ be the solution of the classical RP for (7.1) with initial data $\hat{u}_{L}^{0}, \hat{u}_{R}^{0}$. Denote the 
intermediate state in that solution by $u_{1}^{0}$. Then, the simplified problem in the Toro-Titarev solver for the spatial derivatives is

$$
\frac{\partial}{\partial t} u^{1}+A_{L R} \frac{\partial}{\partial x} u^{1}=0, \quad u^{1}(x, 0)= \begin{cases}\hat{u}_{L}^{1}, & \text { if } x<0 \\ \hat{u}_{R}^{1}, & \text { if } x>0 .\end{cases}
$$

Here, $A_{L R}=A\left(u_{0}^{0}\right)$. We express the vectors $\hat{u}_{L}^{1}, \hat{u}_{R}^{1}$ in terms of the basis $\left\{r_{1}\left(u_{0}^{0}\right)\right.$, $\left.r_{2}\left(u_{0}^{0}\right)\right\}$, i.e.,

$$
\hat{u}_{L}^{1}=\beta_{1} r_{1}\left(u_{0}^{0}\right)+\beta_{2} r_{2}\left(u_{0}^{0}\right), \quad \hat{u}_{R}^{1}=\theta_{1} r_{1}\left(u_{0}^{0}\right)+\theta_{2} r_{2}\left(u_{0}^{0}\right) .
$$

Then, the intermediate state $u_{1}^{1}$ can be computed as

$$
u_{1}^{1}=\theta_{1} r_{1}\left(u_{0}^{0}\right)+\beta_{2} r_{2}\left(u_{0}^{0}\right)=\left(\begin{array}{c}
\left(v_{2}^{1}+w_{2}^{1}\right) \frac{v_{0}^{0}}{v_{0}^{0}+w_{0}^{0}}+\left(v_{0}^{1}+w_{0}^{1}\right) \frac{w_{0}^{0}}{v_{0}^{0}+w_{0}^{0}}-w_{0}^{1} \\
\left(v_{2}^{1}+w_{2}^{1}-v_{0}^{1}-w_{0}^{1}\right) \frac{w_{0}^{0}}{v_{0}^{0}+w_{0}^{0}}+w_{0}^{1}
\end{array}\right) .
$$

Next, we compute the first two terms of the expansion

$$
u(x, t)=q^{0}(\xi)+t q^{1}(\xi)+\ldots .
$$

As above, we find $q^{0}$ by solving the classical RP with initial states $\hat{u}_{L}^{0}, \hat{u}_{R}^{0}$ and we denote the constant states in that solution by $\hat{u}_{L}^{0}=q_{0}^{0}, q_{1}^{0}, q_{2}^{0}=\hat{u}_{R}^{0}$. The function $q^{1}(\xi)$ is given in each domain $D_{i}^{0}$ by

$$
q^{1}(\xi)=\left(\xi-A\left(q_{i}^{0}\right)\right) q_{i}^{1}, \quad 0 \leq i \leq 2,
$$

where we express the unknown vectors $q_{i}^{1}$ as $q_{i}^{1}=\alpha_{i, 1}^{1} r_{1}\left(q_{i}^{0}\right)+\alpha_{i, 2}^{1} r_{2}\left(q_{i}^{0}\right)$.

The coefficients $\alpha_{0, j}^{1}, \alpha_{2, j}^{1}$, for $j=1,2$, are determined by the initial data $\hat{u}_{L}^{1}$ and $\hat{u}_{R}^{1}$ respectively, while the remaining coefficients $\alpha_{1,1}^{1}, \alpha_{1,2}^{2}$ are found by solving a linear $2 \times 2$ system of algebraic equations, as given by Lemma 6.3. We arrive at the system

$$
\begin{aligned}
& \begin{aligned}
&\left(\lambda_{1}\left(q_{1}^{0}\right)-\sigma_{1}^{0}\right)^{2} \ell_{2}\left(q_{0}^{0}\right) \cdot r_{1}\left(q_{1}^{0}\right) \alpha_{1,1}^{1}+\left(\lambda_{2}\left(q_{1}^{0}\right)-\sigma_{1}^{0}\right)^{2} \ell_{2}\left(q_{0}^{0}\right) \\
& \cdot r_{2}\left(q_{1}^{0}\right) \alpha_{1,2}^{1}-\left(\lambda_{2}\left(q_{0}^{0}\right)-\sigma_{1}^{0}\right)^{2} \alpha_{0,2}^{1} \\
&= \frac{\ell_{2}\left(q_{0}^{0}\right) \cdot\left(q_{1}^{0}-q_{0}^{0}\right)}{\ell_{1}\left(q_{0}^{0}\right) \cdot\left(q_{1}^{0}-q_{0}^{0}\right)}\left\{\left(\lambda_{1}\left(q_{1}^{0}\right)-\sigma_{1}^{0}\right)^{2} \ell_{1}\left(q_{0}^{0}\right) \cdot r_{1}\left(q_{1}^{0}\right) \alpha_{1,1}^{1}\right. \\
&\left.+\left(\lambda_{2}\left(q_{1}^{0}\right)-\sigma_{1}^{0}\right)^{2} \ell_{1}\left(q_{0}^{0}\right) \cdot r_{2}\left(q_{1}^{0}\right) \alpha_{1,2}^{1}+\left(\lambda_{1}\left(q_{0}^{0}\right)-\sigma_{1}^{0}\right)^{2} \alpha_{0,1}^{1}\right\}, \\
&\left(\lambda_{1}\left(q_{2}^{0}\right)-\sigma_{2}^{0}\right)^{2} \ell_{1}\left(q_{1}^{0}\right) \cdot r_{1}\left(q_{2}^{0}\right) \alpha_{2,1}^{1}+\left(\lambda_{2}\left(q_{2}^{0}\right)-\sigma_{2}^{0}\right)^{2} \ell_{1}\left(q_{1}^{0}\right) \\
& \quad \cdot r_{2}\left(q_{2}^{0}\right) \alpha_{2,2}^{1}-\left(\lambda_{1}\left(q_{1}^{0}\right)-\sigma_{2}^{0}\right)^{2} \alpha_{1,1}^{1} \\
&=\frac{l_{1}\left(q_{1}^{0}\right) \cdot\left(q_{2}^{0}-q_{1}^{0}\right)}{\ell_{2}\left(q_{1}^{0}\right) \cdot\left(q_{2}^{0}-q_{1}^{0}\right)}\left\{\left(\lambda_{1}\left(q_{2}^{0}\right)-\sigma_{2}^{0}\right)^{2} \ell_{2}\left(q_{1}^{0}\right) \cdot r_{1}\left(q_{2}^{0}\right) \alpha_{2,1}^{1}\right. \\
&\left.\quad+\left(\lambda_{2}\left(q_{2}^{0}\right)-\sigma_{2}^{0}\right)^{2} \ell_{2}\left(q_{1}^{0}\right) \cdot r_{2}\left(q_{2}^{0}\right) \alpha_{2,2}^{1}+\left(\lambda_{2}\left(q_{1}^{0}\right)-\sigma_{2}^{0}\right)^{2} \alpha_{1,2}^{1}\right\} .
\end{aligned} \\
& .7) \quad
\end{aligned}
$$

Now recall that $q_{0}^{0}, q_{1}^{0}, q_{2}^{0}$ are the constant states in the solution of a classical RP. By (7.2)-(7.3) for the intermediate state $q_{1}^{0}$, we find $q_{1}^{0}-q_{0}^{0}=\varepsilon_{1} r_{1}\left(q_{0}^{0}\right)$ and $q_{2}^{0}-q_{1}^{0}=\varepsilon_{2} r_{2}\left(q_{1}^{0}\right)$. Therefore, we get from condition (2.3):

$$
\begin{array}{ll}
\ell_{2}\left(q_{0}^{0}\right) \cdot\left(q_{1}^{0}-q_{0}^{0}\right)=0, & \ell_{1}\left(q_{0}^{0}\right) \cdot\left(q_{1}^{0}-q_{0}^{0}\right)=\varepsilon_{1}, \\
\ell_{1}\left(q_{1}^{0}\right) \cdot\left(q_{2}^{0}-q_{1}^{0}\right)=0, & \ell_{2}\left(q_{1}^{0}\right) \cdot\left(q_{2}^{0}-q_{1}^{0}\right)=\varepsilon_{2} .
\end{array}
$$


Then we can solve (7.6), (7.7) to find

$$
\alpha_{1,1}^{1}=-\left(v_{2}^{1}+w_{2}^{1}\right) \frac{\sqrt{\left(v_{1}^{0}\right)^{2}+\left(w_{1}^{0}\right)^{2}}}{v_{2}^{0}+w_{2}^{0}}, \quad \alpha_{1,2}^{1}=\sqrt{2}\left(\frac{v_{2}^{0}+w_{2}^{0}}{v_{0}^{0}+w_{0}^{0}}\right)^{2}\left(\left(v_{0}^{1}+w_{0}^{1}\right) \frac{w_{0}^{0}}{v_{0}^{0}+w_{0}^{0}}-w_{0}^{1}\right) .
$$

Thus, the coefficient $q_{1}^{1}$ is given by

$q_{1}^{1}=\alpha_{1,1}^{1} r_{1}\left(q_{1}^{0}\right)+\alpha_{1,2}^{1} r_{2}\left(q_{1}^{0}\right)=\left(\begin{array}{l}\left(v_{2}^{1}+w_{2}^{1}\right) \frac{v_{0}^{0}}{v_{0}^{0}+w_{0}^{0}}+\left(\frac{v_{2}^{0}+w_{2}^{0}}{v_{0}^{0}+w_{0}^{0}}\right)^{2}\left(\left(v_{0}^{1}+w_{0}^{1}\right) \frac{w_{0}^{0}}{v_{0}^{0}+w_{0}^{0}}-w_{0}^{1}\right) \\ \left(v_{2}^{1}+w_{2}^{1}\right) \frac{v_{0}^{0}}{v_{0}^{0}+w_{0}^{0}}-\left(\frac{v_{2}^{0}+w_{2}^{0}}{v_{0}^{0}+w_{0}^{0}}\right)^{2}\left(\left(v_{0}^{1}+w_{0}^{1}\right) \frac{w_{0}^{0}}{v_{0}^{0}+w_{0}^{0}}-w_{0}^{1}\right)\end{array}\right)$.

The only difference between $u_{1}^{1}$ and $q_{1}^{1}$ is the factor $\left(\left(v_{2}^{0}+w_{2}^{0}\right) /\left(v_{0}^{0}+w_{0}^{0}\right)\right)^{2}$ whose size only depends on the distance of the states $\hat{u}_{L}^{0}$ and $\hat{u}_{R}^{0}$.

7.2. Shallow water equations. We consider the GRP for the shallow water equations,

$$
\frac{\partial}{\partial t}\left(\begin{array}{c}
h \\
h u
\end{array}\right)+\frac{\partial}{\partial x}\left(\begin{array}{c}
h u \\
h u^{2}+\frac{1}{2} g h^{2}
\end{array}\right)=0
$$

where $g$ is a constant, with initial data $\hat{h}_{L}=\hat{h}_{R}=1$ and

$$
\hat{u}_{L}(x)=a_{L} x^{2}+b_{L} x+c_{L}, \quad \hat{u}_{R}(x)=a_{R} x^{2}+b_{R} x+c_{R} .
$$

When $c_{R}<0<c_{L}$, this data leads to a solution with two shock waves.

We compare the resulting approximations up to terms of second order obtained by the LeFloch-Raviart expansion and the Toro-Titarev solver, respectively. Reference solutions are obtained by a random choice method (RCM) on a very fine grid using an exact Riemann solver and van der Corput pseudo random numbers (see [32, Chapter 7] for details). We perform two series of tests:

(i) Large jumps in the initial data, fixed derivatives. We fix $a_{L}=0.02, a_{R}=$ -0.01 and $b_{L}=0.4, b_{R}=-0.2$. We solve the GRP for $c_{R}=-1$ and $c_{L}=0,2,4$, respectively. Results are shown in Figure 1 Denoting $v=h$ and $w=h u$, the plots show the reference solution (thick black line), the LeFloch-Raviart approximation (circles) and the Toro-Titarev approximation (crosses) along the line $\xi=0$ for times $0 \leq t \leq 0.05$. The difference in the two approximations increases with the size of the jump. We observe that the LeFloch-Raviart approximation is almost identical to the reference solution.

(ii) Large jumps in the derivatives, fixed jump in states. We fix $a_{L}=$ $0.02, a_{R}=-0.01$ and $c_{L}=0.2, c_{R}=-0.2$, so we have a fixed jump $\left|\hat{u}_{L}^{0}-\hat{u}_{R}^{0}\right|=0.4$. We let $b_{R}=-1$ and test for different values of $b_{L}$; see Figure 2, For all test cases both approximations are very close to the reference solution.

We conclude that the Toro-Titarev solver gives a very good approximation when the jump in the initial data is small (independent of the jumps in derivatives), but it introduces a larger error when the jump in the states is large. Note that this behaviour is consistent with our analysis, see the remark after Theorem 6.4 


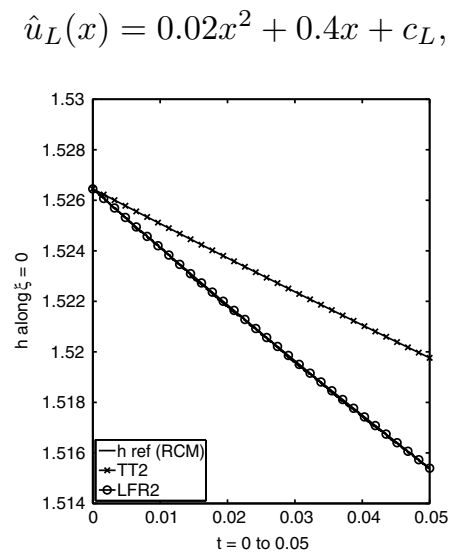

$\hat{u}_{R}(x)=-0.01 x^{2}-0.2 x-1$

(a) $c_{L}=2, \quad\left|\hat{u}_{L}^{0}-\hat{u}_{R}^{0}\right|=3$
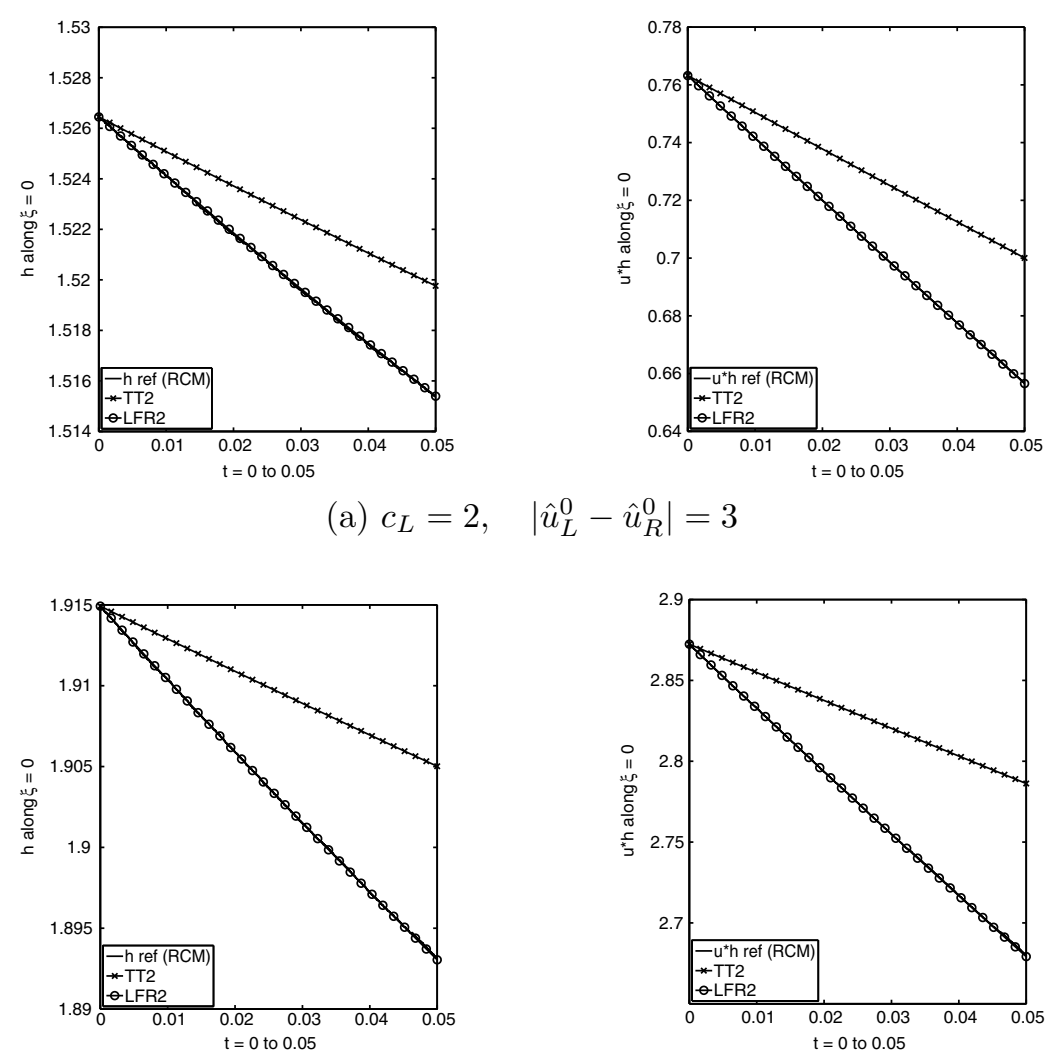

(b) $c_{L}=4, \quad\left|\hat{u}_{L}^{0}-\hat{u}_{R}^{0}\right|=5$
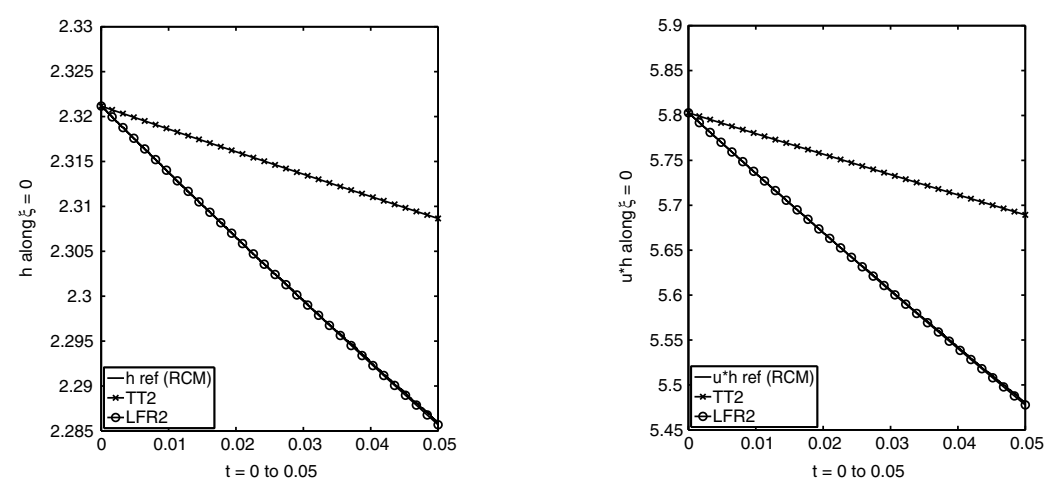

(c) $c_{L}=6, \quad\left|\hat{u}_{L}^{0}-\hat{u}_{R}^{0}\right|=7$

Figure 1. Shallow water GRP with two shocks: Jumps in the initial states. 

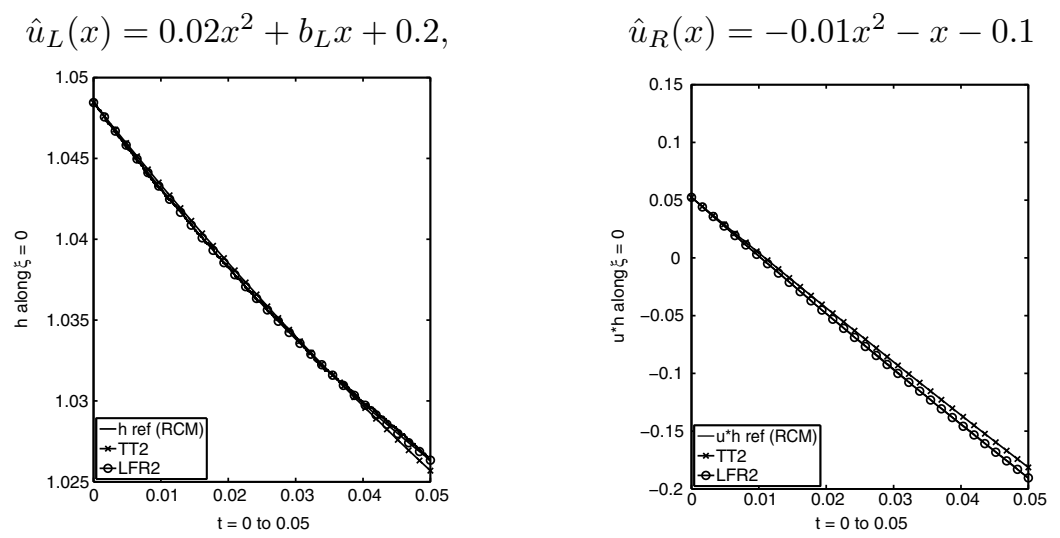

(a) $b_{L}=2, \quad\left|\hat{u}_{L}^{1}-\hat{u}_{R}^{1}\right|=3$
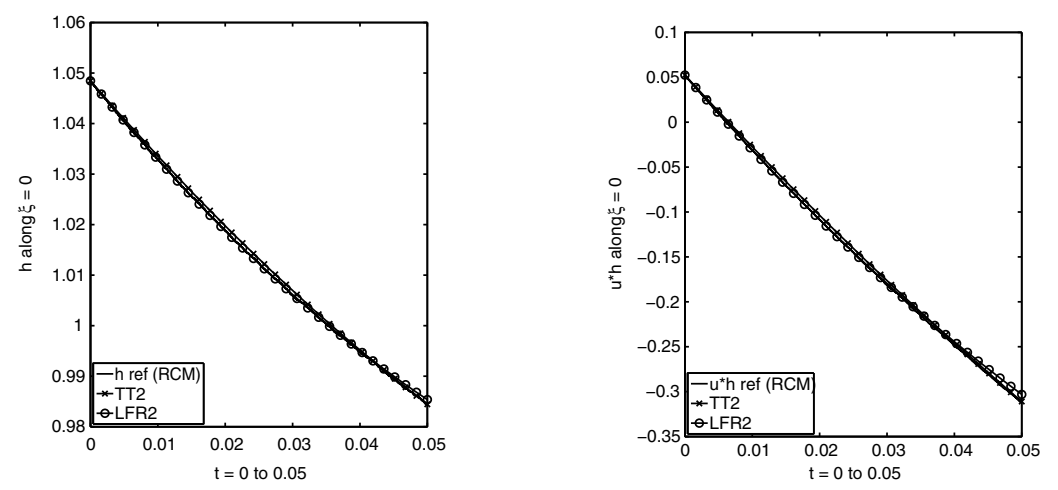

(b) $b_{L}=4, \quad\left|\hat{u}_{L}^{1}-\hat{u}_{R}^{1}\right|=5$
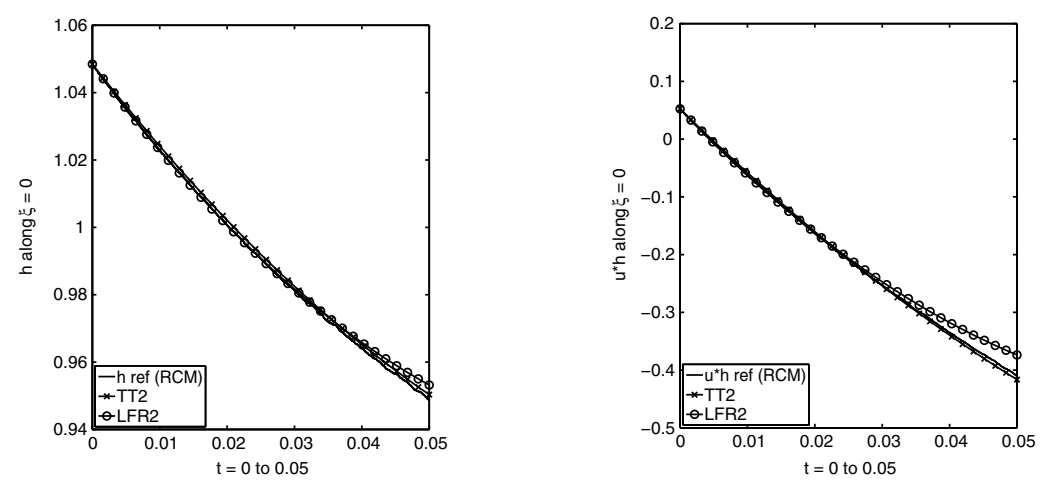

(c) $b_{L}=6, \quad\left|\hat{u}_{L}^{1}-\hat{u}_{R}^{1}\right|=7$

FiguRE 2. Shallow water GRP with two shocks: Jumps in the initial derivatives. 
$\hat{s}_{L}(x)=0.1 x^{2}+0.1 x+0.5$,

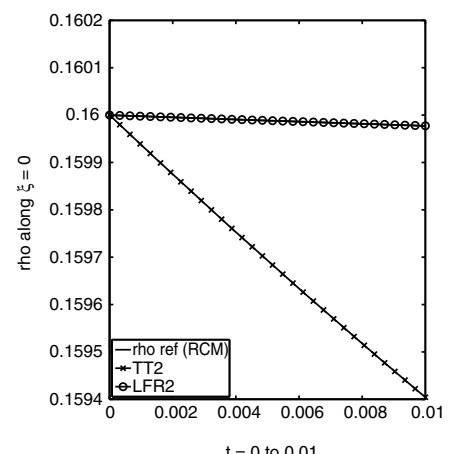

(a) $c_{R}=33 / 6, \quad\left|\hat{u}_{L}^{0}-\hat{u}_{R}^{0}\right|_{1}=3$
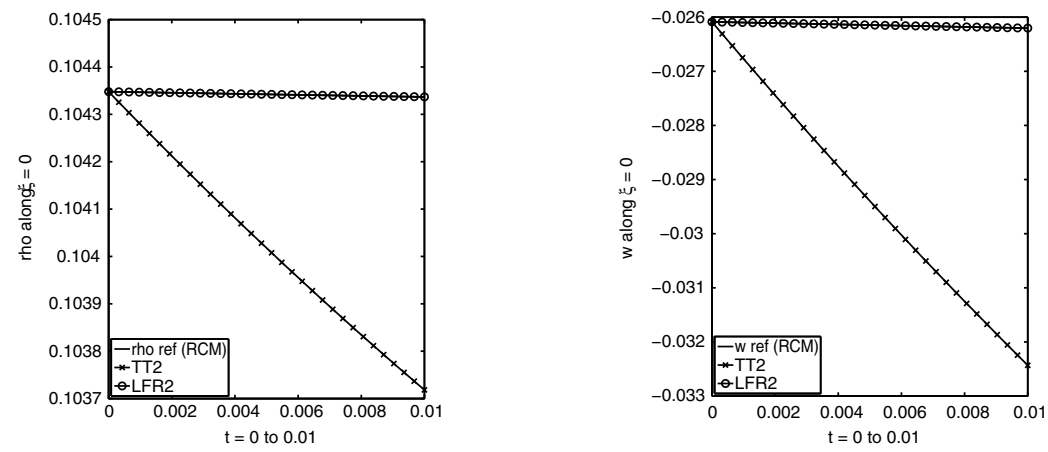

(b) $c_{R}=53 / 6, \quad\left|\hat{u}_{L}^{0}-\hat{u}_{R}^{0}\right|_{1}=5$
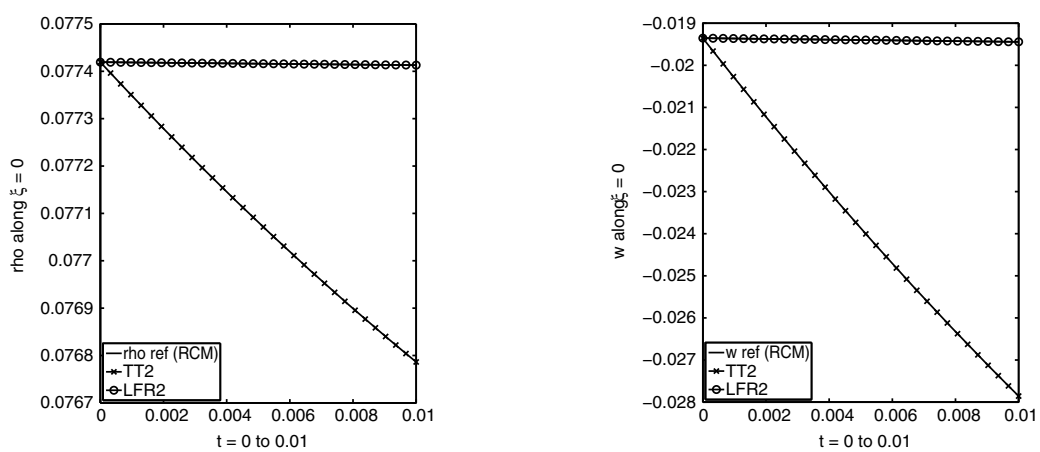

(c) $c_{R}=73 / 6, \quad\left|\hat{u}_{L}^{0}-\hat{u}_{R}^{0}\right|_{1}=7$

Figure 3. Aw-Rascle type traffic flow: Jumps in the initial states. 
$\hat{s}_{L}(x)=0.5 x^{2}+0.1 x+1.1129$,

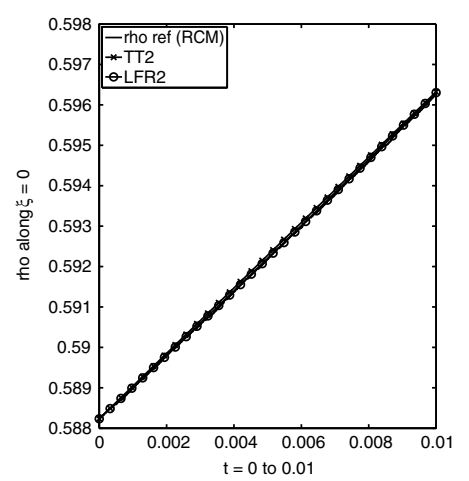

$\hat{s}_{R}(x)=-0.1 x^{2}+b_{R} x+1.2$

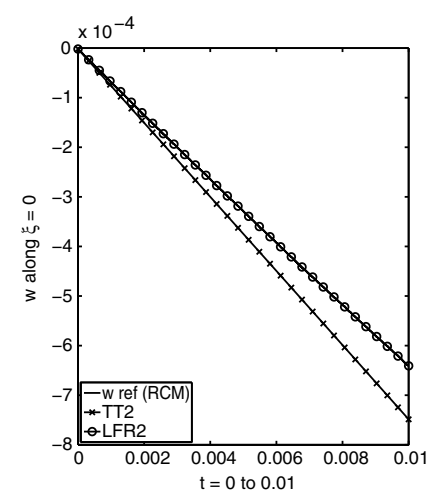

(a) $b_{R}=-4.833, \quad\left|\hat{u}_{L}^{1}-\hat{u}_{R}^{1}\right|_{1}=3$
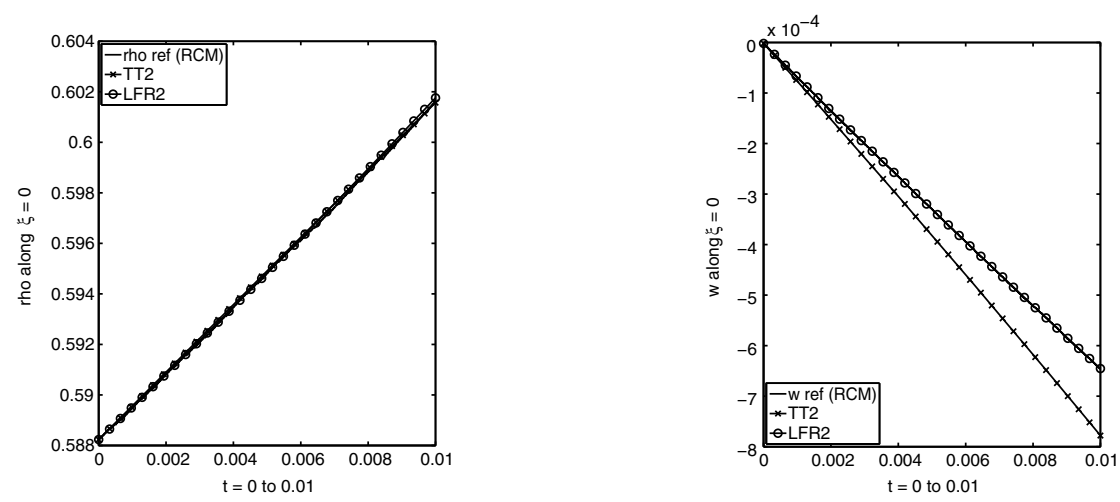

(b) $b_{R}=-7.8167, \quad\left|\hat{u}_{L}^{1}-\hat{u}_{R}^{1}\right|_{1}=5$
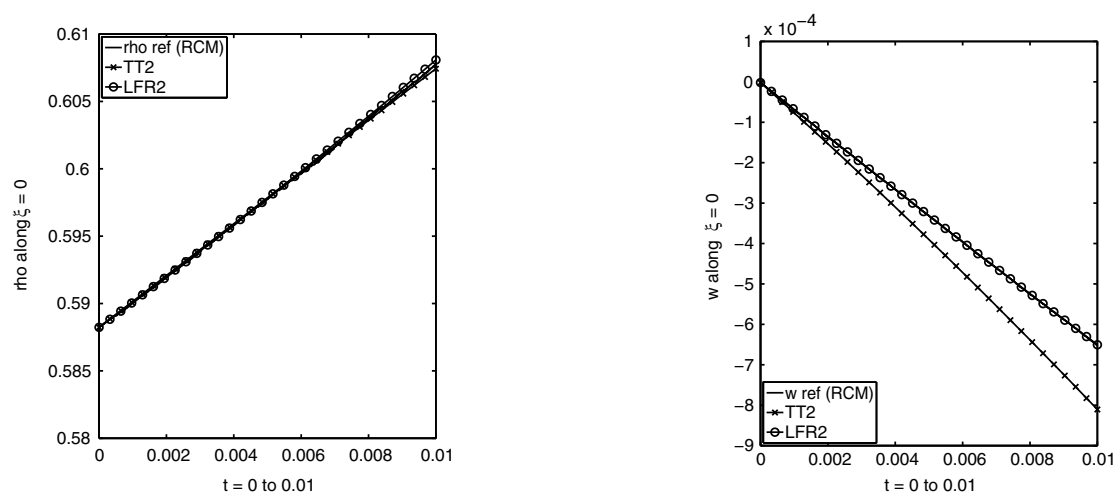

(c) $b_{R}=-11.15, \quad\left|\hat{u}_{L}^{1}-\hat{u}_{R}^{1}\right|_{1}=7$

Figure 4. Aw-Rascle type traffic flow: Jumps in initial first derivatives. 
7.3. A system from traffic flow. We study a variant of a macroscopic model of traffic flow that was proposed by Aw and Rascle 2. We consider the system

$$
\begin{aligned}
\frac{\partial}{\partial t} \rho+\frac{\partial}{\partial x}(\rho s) & =0 \\
\frac{\partial}{\partial t}(s+p(\rho))+s \frac{\partial}{\partial x}(s+p(\rho)) & =0 .
\end{aligned}
$$

Here, $\rho$ is the density of cars, $s$ the velocity. The function $p$ acts like a pressure term in fluid dynamics, but is understood as an 'anticipation factor'. Global existence and smoothness of the solution to a GRP for (7.8) are discussed in [27. We assume $\rho>0$ (although the case $\rho=0$ can be handled) and we also assume $p^{\prime}(\rho)>0$, where the specific choice of $p$ will be discussed later.

Setting

$$
w=\rho(s+p(\rho))
$$

the system can be written as

$$
\begin{aligned}
\frac{\partial}{\partial t} \rho+\frac{\partial}{\partial x}(\rho s) & =0 \\
\frac{\partial}{\partial t} w+\frac{\partial}{\partial x}(w s) & =0 .
\end{aligned}
$$

Noting that $s=w / \rho-p(\rho)$ we let $u=(\rho, w)^{T}$ and arrive at

$$
\frac{\partial}{\partial t} u+\frac{\partial}{\partial x} f(u)=0, \quad f(u)=\left(\begin{array}{c}
w-\rho p(\rho) \\
w^{2} / \rho-w p(\rho)
\end{array}\right) .
$$

Then the eigenvalues and eigenvectors of $A=D f(u)$ are

$$
\begin{aligned}
\lambda_{1}(\rho, w)=\frac{w}{\rho}-p(\rho)-\rho p^{\prime}(\rho), & r_{1}(\rho, w) & =\left(\begin{array}{c}
1 \\
\frac{w}{\rho}
\end{array}\right), \\
\lambda_{2}(\rho, w)=\frac{w}{\rho}-p(\rho)=s, & r_{2}(\rho, w) & =\left(\begin{array}{c}
1 \\
\frac{w}{\rho}+\rho p^{\prime}(\rho)
\end{array}\right) .
\end{aligned}
$$

It is easy to see that the second field is always linearly degenerate, while the first field is linearly degenerate, if and only if

$$
p(\rho)=a-\frac{b}{\rho}, \quad a, b>0 .
$$

Under this assumption, both waves are contact discontinuities. For actually modelling traffic flow, one usually takes $p$ in the form $p(\rho)=\rho^{\gamma}, \gamma>0$. However, for purposes of analysis and less of modelling, we are interested in a nonlinear system for which all fields are linearly degenerate. With this choice of $p$ we present some numerical results considering the GRP with piecewise constant density $\hat{\rho}_{L}$ and $\hat{\rho}_{R}$, and piecewise quadratic velocity $\hat{s}_{L}, \hat{s}_{R}$. We fix $a=0.5, b=1$.

As in the previous section, we compare the resulting approximations up to quadratic terms obtained by the Toro-Titarev solver and the LeFloch-Raviart solver, respectively. Reference solutions are, again, obtained by a random choice method 
(RCM) on a very fine grid using an exact Riemann solver and van der Corput pseudo random numbers. We perform two series of tests:

(i) Large jumps in the initial data, fixed derivatives. We take

$$
\hat{\rho}_{L}(x)=0.8, \quad \hat{\rho}_{R}(x)=0.6
$$

and solve the generalized Riemann problem for different choices of $\hat{s}_{L}, \hat{s}_{R}$. Results along the line $\xi=0$ for $0 \leq t \leq 0.01$ are shown in Figure 3 .

(ii) Large jumps in first derivatives, fixed jump in states We take

$$
\hat{\rho}_{L}(x)=0.62, \quad \hat{\rho}_{R}(x)=0.6
$$

and choose different $\hat{s}_{L}, \hat{s}_{R}$, such that we have small jumps in the initial states and large jumps in first derivatives; see Figure 4 .

We observe the same qualitative behaviour as for the shallow water equations: When the size of the jump in the initial data is small, both methods give good results, even for large jumps in the derivatives. The approximation obtained by the LeFloch-Raviart solver is in all cases very close to the reference solution. We observe further that the Toro-Titarev solver is highly sensitive towards jumps in the initial data and in the first series of tests (see Figure 3) the results obtained with the Toro-Titarev solver differ largely from the reference solution. While this is consistent with our analytical results, the size of the error seems counterintuitive. In the case of linear systems the Toro-Titarev solver reproduces the Taylor expansion exactly, so one might expect a good approximation for a system with only linearly degenerate fields. However, a detailed study of the effects of linearly degenerate fields on the quality of approximate GRP solvers is beyond the scope of this paper.

\section{REFERENCES}

[1] T. Aboiyar, E. H. Georgoulis, and A. Iske, Adaptive ADER methods using kernel-based polyharmonic spline WENO reconstruction, SIAM J. Sci. Comput. 32 (2010), no. 6, 3251-3277, DOI 10.1137/100792573. MR2746620(2012a:65217)

[2] A. Aw and M. Rascle, Resurrection of "second order" models of traffic flow, SIAM J. Appl. Math. 60 (2000), no. 3, 916-938 (electronic), DOI 10.1137/S0036139997332099. MR1750085 (2001a:35111)

[3] D. S. Balsara and C.-W. Shu, Monotonicity preserving weighted essentially non-oscillatory schemes with increasingly high order of accuracy, J. Comput. Phys. 160 (2000), no. 2, 405452, DOI 10.1006/jcph.2000.6443. MR1763821

[4] M. Ben-Artzi and J. Falcovitz, A second-order Godunov-type scheme for compressible fluid dynamics, J. Comput. Phys. 55 (1984), no. 1, 1-32, DOI 10.1016/0021-9991(84)90013-5. MR757422 (86f:65146)

[5] Matania Ben-Artzi and Joseph Falcovitz, Generalized Riemann Problems in Computational Fluid Dynamics, Cambridge University Press, Cambridge, 2003.

[6] A. Bourgeade, Ph. LeFloch, and P.-A. Raviart, An asymptotic expansion for the solution of the generalized Riemann problem. II. Application to the equations of gas dynamics (English, with French summary), Ann. Inst. H. Poincaré Anal. Non Linéaire 6 (1989), no. 6, 437-480. MR.1035338(91c:35087)

[7] A. Bressan, Hyperbolic Systems of Conservation Laws: The One-Dimensional Cauchy Problem, Oxford Lecture Series in Mathematics and its Applications, vol. 20, Oxford University Press, Oxford, 2000. MR 1816648(2002d:35002)

[8] C. E. Castro and E. F. Toro, Solvers for the high-order Riemann problem for hyperbolic balance laws, J. Comput. Phys. 227 (2008), no. 4, 2481-2513, DOI 10.1016/j.jcp.2007.11.013. MR2388072(2009a:65196) 
[9] S. Chen, X. Han, and H. Zhang, The generalized Riemann problem for first order quasilinear hyperbolic systems of conservation laws. II, Acta Appl. Math. 108 (2009), no. 2, 235-277, DOI 10.1007/s10440-008-9311-6. MR2551474 (2010j:35311)

[10] S. Chen, D. Huang, and X. Han, The generalized Riemann problem for first order quasilinear hyperbolic systems of conservation laws. I, Bull. Korean Math. Soc. 46 (2009), no. 3, 409-434, DOI 10.4134/BKMS.2009.46.3.409. MR2522855 (2010f:35239)

[11] P. Woodward and P. Colella, The numerical simulation of two-dimensional fluid flow with strong shocks, J. Comput. Phys. 54 (1984), no. 1, 115-173, DOI 10.1016/0021-9991(84)901426. MR748569 (85e:76004)

[12] E. Harabetian, A convergent series expansion for hyperbolic systems of conservation laws, Trans. Amer. Math. Soc. 294 (1986), no. 2, 383-424, DOI 10.2307/2000190. MR825712 (87k:35160)

[13] A. Harten, B. Engquist, S. Osher, and S. R. Chakravarthy, Uniformly high-order accurate essentially nonoscillatory schemes. III, J. Comput. Phys. 71 (1987), no. 2, 231-303, DOI 10.1016/0021-9991(87)90031-3. MR897244(90a:65199)

[14] G.-S. Jiang and C.-W. Shu, Efficient implementation of weighted ENO schemes, J. Comput. Phys. 126 (1996), no. 1, 202-228, DOI 10.1006/jcph.1996.0130. MR1391627 (97e:65081)

[15] M. Käser and A. Iske, ADER schemes on adaptive triangular meshes for scalar conservation laws, J. Comput. Phys. 205 (2005), no. 2, 486-508, DOI 10.1016/j.jcp.2004.11.015. MR2134991(2005m:65172)

[16] V. P. Kolgan, Application of the principle of minimizing the derivative to the construction of finite-difference schemes for computing discontinuous solutions of gas dynamics, J. Comput. Phys. 230 (2011), no. 7, 2384-2390, DOI 10.1016/j.jcp.2010.12.033. Translated from the Russian by Konstantin Kabin and Valeriy Tenishev. MR2772921 (2012a:76109)

[17] D.-X. Kong, Global structure stability of Riemann solutions of quasilinear hyperbolic systems of conservation laws: shocks and contact discontinuities, J. Differential Equations 188 (2003), no. 1, 242-271, DOI 10.1016/S0022-0396(02)00068-2. MR1954515 (2004b:35222)

[18] D.-X. Kong, Global structure instability of Riemann solutions of quasilinear hyperbolic systems of conservation laws: rarefaction waves, J. Differential Equations 219 (2005), no. 2, 421-450, DOI 10.1016/j.jde.2005.03.001. MR2183267 (2006i:35236)

[19] P. D. Lax, Hyperbolic systems of conservation laws. II, Comm. Pure Appl. Math. 10 (1957), 537-566. MR0093653 (20 \#176)

[20] Ph. LeFloch and P.-A. Raviart, An asymptotic expansion for the solution of the generalized Riemann problem. I. General theory (English, with French summary), Ann. Inst. H. Poincaré Anal. Non Linéaire 5 (1988), no. 2, 179-207. MR954470 (90a:35140)

[21] P. G. LeFloch and T. T. Li, A global in time asymptotic expansion for the solution of the generalized Riemann problem, Asymp. Analysis 3 (1991), 321-340.

[22] T. T. Li and W. C. Yu, Boundary value problems for quasilinear hyperbolic systems, Duke University Mathematics Series, V, Duke University, Mathematics Department, Durham, NC, 1985. MR823237 (88g:35115)

[23] X.-D. Liu, S. Osher, and T. Chan, Weighted essentially non-oscillatory schemes, J. Comput. Phys. 115 (1994), no. 1, 200-212, DOI 10.1006/jcph.1994.1187. MR1300340

[24] I. S. Men'shov, Increase in the approximation order for Godunov's scheme on the basis of the solution of the generalized Riemann problem (Russian), Zh. Vychisl. Mat. i Mat. Fiz. 30 (1990), no. 9, 1357-1371, 1439; English transl., U.S.S.R. Comput. Math. and Math. Phys. 30 (1990), no. 5, 54-65 (1991). MR.1085016 (91m:76093)

[25] T. Schwartzkopff, C. D. Munz, and E. F. Toro, ADER: a high-order approach for linear hyperbolic systems in 2D, Proceedings of the Fifth International Conference on Spectral and High Order Methods (ICOSAHOM-01) (Uppsala), J. Sci. Comput. 17 (2002), no. 1-4, 231240, DOI 10.1023/A:1015160900410. MR1910564

[26] J. Smoller, Shock waves and reaction-diffusion equations, Grundlehren der Mathematischen Wissenschaften [Fundamental Principles of Mathematical Science], vol. 258, Springer-Verlag, New York-Berlin, 1983. MR688146 (84d:35002)

[27] T. Li and L. Wang, Global propagation of regular nonlinear hyperbolic waves, Progress in Nonlinear Differential Equations and their Applications, 76, Birkhäuser Boston, Inc., Boston, MA, 2009. MR2524566 (2012b:35002)

[28] B. Temple, Systems of conservation laws with invariant submanifolds, Trans. Amer. Math. Soc. 280 (1983), no. 2, 781-795, DOI 10.2307/1999646. MR716850(84m:35080) 
[29] V. A. Titarev and E. F. Toro, ADER: arbitrary high order Godunov approach, Proceedings of the Fifth International Conference on Spectral and High Order Methods (ICOSAHOM01) (Uppsala), J. Sci. Comput. 17 (2002), no. 1-4, 609-618, DOI 10.1023/A:1015126814947. MR.1910755

[30] V. A. Titarev and E. F. Toro, ADER schemes for three-dimensional non-linear hyperbolic systems, J. Comput. Phys. 204 (2005), no. 2, 715-736, DOI 10.1016/j.jcp.2004.10.028. MR2131859(2005k:65183)

[31] V. A. Titarev and E. F. Toro, Analysis of ADER and ADER-WAF schemes, IMA J. Numer. Anal. 27 (2007), no. 3, 616-630, DOI 10.1093/imanum/drl033. MR2337583 (2008e:65268)

[32] E. F. Toro, Riemann solvers and numerical methods for fluid dynamics, 3rd ed., SpringerVerlag, Berlin, 2009. A practical introduction. MR2731357 (2011i:76059)

[33] E. F. Toro and A. Hidalgo, ADER finite volume schemes for nonlinear reaction-diffusion equations, Appl. Numer. Math. 59 (2009), no. 1, 73-100, DOI 10.1016/j.apnum.2007.12.001. MR2474104(2009j:65212)

[34] E. F. Toro, R. C. Millington, and L. A. M. Nejad, Towards very high order Godunov schemes, Godunov methods (Oxford, 1999), Kluwer/Plenum, New York, 2001, pp. 907-940. MR.1963645(2003m:65151)

[35] E. F. Toro and V. A. Titarev, ADER schemes for scalar non-linear hyperbolic conservation laws with source terms in three-space dimensions, J. Comput. Phys. 202 (2005), no. 1, 196215, DOI 10.1016/j.jcp.2004.06.014. MR2102882 (2005h:65140)

[36] E. F. Toro and V. A. Titarev, TVD fluxes for the high-order ADER schemes, J. Sci. Comput. 24 (2005), no. 3, 285-309, DOI 10.1007/s10915-004-4790-8. MR2219366 (2006m:65174)

[37] E. F. Toro and V. A. Titarev, Derivative Riemann solvers for systems of conservation laws and ADER methods, J. Comput. Phys. 212 (2006), no. 1, 150-165, DOI 10.1016/j.jcp.2005.06.018. MR2183608 (2007b:65077)

[38] B. van Leer, Towards the ultimate conservative difference scheme. $V$ : A second order sequel to Godunov's method, J. Comput. Phys. 32 (1979), no. 1, 101-136.

Department of Civil, Environmental and Mechanical Engineering, University of Trento, via Mesiano 77, I-38123 Trento, Italy

E-mail address: clausruediger.goetz@unitn.it

Department of Mathematics, University of Hamburg, Bundesstr. 55, D-20146 HamBURG, GERMANY

E-mail address: armin.iske@uni-hamburg.de 